\title{
Proton-triton nuclear reaction in ICRF heated plasmas in JET
}

\author{
M I K Santala ${ }^{1}$, M J Mantsinen ${ }^{1}$, L Bertalot ${ }^{2}$, S Conroy ${ }^{3}$, \\ V Kiptily ${ }^{4}$, S Popovichev ${ }^{4}$, A Salmi $^{1}$, D Testa ${ }^{5}$, Yu Baranov ${ }^{4}$, \\ P Beaumont ${ }^{4}, \mathbf{P}$ Belo $^{6}$, J Brzozowski ${ }^{7}, \mathrm{M}_{\text {Cecconello }}{ }^{7}$, \\ M deBaar ${ }^{8}, \mathbf{P}$ deVries $^{4}, \mathrm{C}_{\text {Gowers }}{ }^{4}, \mathbf{J}-\mathrm{M}$ Noterdaeme $^{9,10}$, \\ C Schlatter ${ }^{5}$, S Sharapov ${ }^{4}$, and JET-EFDA contributorsł \\ ${ }^{1}$ Helsinki University of Technology, Association Euratom-Tekes, FI-02015 TKK, \\ Finland \\ ${ }^{2}$ Associazione Euratom/ENEA/CNR sulla Fusione, Frascati, Via Enrico Fermi 45, \\ C.P. 65, I-00044 Frascati (Rome), Italy \\ ${ }^{3}$ INF, EURATOM/VR Association, Ångström Laboratory, Uppsala University, \\ Box 525, SE-75120 Uppsala, Sweden \\ ${ }^{4}$ EURATOM/UKAEA Fusion Association, Culham Science Centre, Abingdon, \\ Oxon, OX14 3DB, United Kingdom \\ ${ }^{5}$ CRPP, Association EURATOM - Confédération Suisse, EPFL, CH-1015 Lausanne, \\ Switzerland \\ ${ }^{6}$ Centro de Fusão Nuclear, Associação EURATOM-IST, Instituto Superior Técnico, \\ Av. Rovisco Pais, 1049-001 Lisboa, Portugal \\ ${ }^{7}$ Alfvén Laboratory, Association EURATOM-VR, Royal Institute of Technology, \\ SE-10044 Stockholm, Sweden \\ ${ }^{8}$ FOM-Instituut voor Plasmafysica, Association Euratom-FOM, TEC, PO Box 1207, \\ NL-3430 BE Nieuwegein, The Netherlands \\ ${ }^{9}$ Max-Planck IPP-EURATOM Assoziation, Boltzmann-Str. 2, D-85748 Garching b. \\ München, Germany \\ ${ }^{10}$ Gent University, EESA Department, St. Pietersnieuwstraat 41, B-9000 Gent, \\ Belgium
}

\begin{abstract}
Fast protons can react with tritons in an endothermic nuclear reaction which can act as a source of neutrons in magnetically confined fusion plasmas. We have performed an experiment to systematically study this reaction in low tritium concentration $(\approx 1 \%)$ plasmas in the Joint European Torus $(\mathrm{JET})$. A linear dependence is found between excess neutron rate and tritium concentration when the DT fusion rate is low. We discuss the properties of the neutron emission, including anisotropy, from the proton-triton reaction in a fusion reactor environment and derive simple models for the calculation of the neutron yield from this reaction in terms of tritium density, fast ion temperature and fast ion energy content.
\end{abstract}

PACS numbers: $52.70 . \mathrm{Nc}, 52.70 . \mathrm{La}, 52.25 . \mathrm{Tx}, 52.55 . \mathrm{Fa}$

$\ddagger$ See the Appendix of J.Pamela et al., Fusion Energy 2004 (Proc. 20th Int. Conf. Vilamoura, 2004) IAEA, Vienna (2004). 
E-mail: Marko.Santala@hut.fi 


\section{Introduction}

In magnetically confined fusion plasmas, other nuclear reactions can take place in addition to the main D-T, D-D and D- ${ }^{3} \mathrm{He}$ fusion reactions. This is especially true, when plasmas contain ion populations with highly supra-thermal energy distributions. Such populations can be produced, for example, by fusion reactions (fusion product ions) or knock-on collisions with fusion product ions and by RF-heating of the plasma ions.

The endothermic reaction between energetic protons with background tritons (sometimes called "pT-fusion"), $\mathrm{T}+p \rightarrow n+{ }^{3} \mathrm{He}+Q$ with $Q=-764 \mathrm{keV}$ (calculated from [1]), could in some cases act as a significant source of neutrons. Fast protons are often present in RF-heated plasmas, and the reaction has a large cross-section $\left(\sigma_{\mathrm{pT}}\right)$ above $E_{p}>1019 \mathrm{keV}$, its threshold $\left(E_{\mathrm{th}, \text { lab }}\right)$ for tritons at rest (Fig. 1). If the pT reaction rate can be accurately measured, it could possibly used as an additional fast ion diagnostic. If the proton tail in plasma is energetic enough, it becomes possible to induce other neutron-producing nuclear reactions, like with ${ }^{9} \mathrm{Be}(Q=-1850 \mathrm{keV}$, calculated from [1]), a common impurity ion in JET.

In prior fusion-related work, the pT reaction has been observed in JET in Hminority ICRF-heated plasmas with high tritium concentration $\left(n_{t} / n_{e}>0.9\right)$ [2]. In that experiment, the total neutron rate was up to $40 \%$ more than the rate attributable to D-T fusion reactions. However, a more detailed study of this reaction was not possible at that time.

In this paper, we report on a recent experimental study of the pT reaction done on the JET machine using hydrogen minority ICRF-heated plasmas with low tritium concentration (order of $1 \%$ ) in the TTE (Trace Tritium Experiment) campaign. We have varied the hydrogen and tritium concentration as well as the RF heating scheme (monochromatic vs. polychromatic, i. e. single-frequency vs. multifrequency) and RF power to assess their impact on the pT neutron production. We have used excess neutron production as an indicator for $\mathrm{pT}$ reaction, taking into account other potential sources that could be contributing to this excess. We make a preliminary assessment of the significance of the $\mathrm{pT}$ reaction for future JET tritium campaigns.

In the first section of this paper we discuss the pT reaction, especially the kinematics and how to detect the reaction. In the second section we model the $\mathrm{pT}$ yield using a simple, generic analytic model and by PION [10] computer simulations. The third section introduces the experiment that has been carried out and the obtained results are presented in the fourth section. Finally, we discuss the importance of pT reaction in JET and future fusion experiments.

\subsection{Kinematics of $p T$ reaction}

The $\mathrm{pT}$ reaction and its exothermic mirror reaction ${ }^{3} \mathrm{He}+n \rightarrow p+\mathrm{T}$ have been extensively studied due to their importance in cosmology and as neutron detection and production reactions $[3,4]$. As is typical for high-energy nuclear reactions, the 


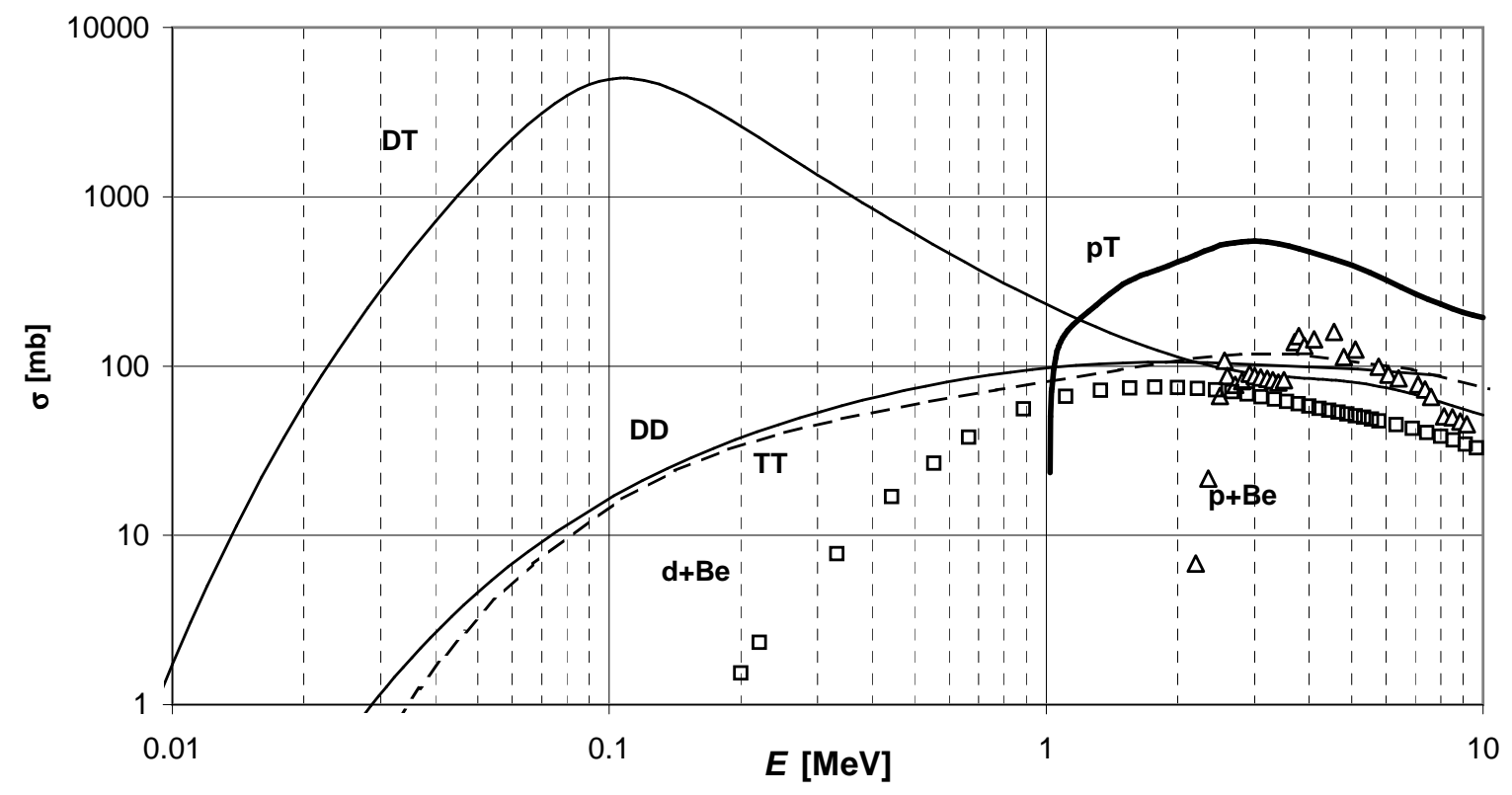

Figure 1. Microscopic reaction cross-sections for the pT reaction [4] and other neutron-producing fusion reactions (DT, DD and TT)[5]. $x$-axis is the energy of the projectiles, interacting with stationary targets. Note the sharp threshold of the $\mathrm{pT}$ reaction at $1 \mathrm{MeV}$ and the large cross section above that. Impurity reactions of deuterons and protons with ${ }^{9} \mathrm{Be}$ are also shown. [4]

kinematics of the pT reaction is highly anisotropic. Here we analyse and assess the impact of this anisotropy in magnetic fusion environment. Initially, we assume tritons at rest, however, we also show that the thermal motion of tritons has very little impact.

The kinematic anisotropy in $\mathrm{pT}$ reaction is significant because the motion of the centre-of-mass (CM) is important for the motion of the reaction products. This is because a high-energy proton is required to induce the reaction but much of the initial energy is lost in the endothermic reaction. In the CM frame, the excess energy remaining after the $\mathrm{pT}$ reaction is split according to inverse particle masses, $3 / 4$ for the neutron $\left(E_{n, \mathrm{CM}}\right)$ and $1 / 4$ for the helium nucleus. To find out the energy and angular distributions in the lab frame, the motion of the CM frame must be added to the particle motion in the CM frame, resulting in large anisotropy in the neutron energy in the lab frame (Fig. 2a): If $E_{p} \gg E_{\text {th,lab }}$ the neutron energy in the lab frame $E_{n, \text { lab }} \approx E_{p, \text { lab }}-Q$ in the forward direction and $E_{n, \text { lab }} \approx E_{p, \text { lab }} / 4$ in the backward direction. The asymmetry is larger with lower $E_{p} . \S$ From kinematics only, it follows that scattering to forward angles in the lab frame is preferred.

The pT reaction is generally anisotropic also in CM frame, in a manner varying strongly with energy. Combining the anisotropy in the CM frame with the purely

$\S$ Close to threshold (below $1147 \mathrm{keV}$ ), double-valued emission in forward-direction results because the neutron velocity in the CM frame is below the velocity of the CM. Although this is not likely to be important for overall observables in magnetic plasma environment, such a regime may cause difficulties in numerical simulations. 

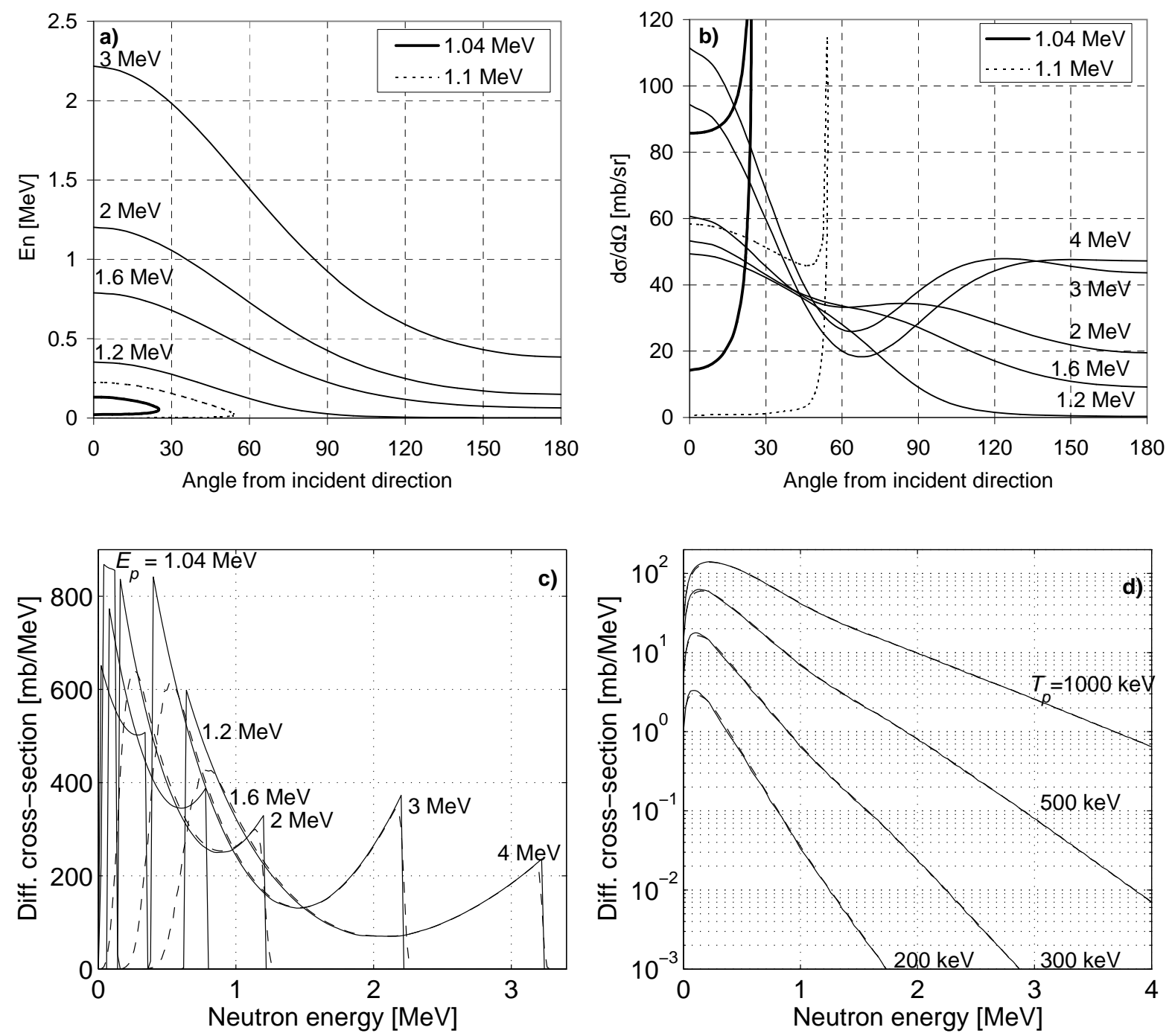

Figure 2. Kinematics of the pT reaction in cold and warm plasma. a) The energy of the produced neutron $E_{n}$ vs. the direction in the lab frame for selected incident proton energies (labels). b) Differential reaction cross-section $d \sigma / d \Omega$ for selected proton energies in the lab frame. Due to kinematics and energy-dependent anisotropy in the CM frame $d \sigma / d \Omega$ has strong angular dependence, generally favouring forwardscattering, and the shape depends strongly on proton energy. a) and b) are plotted assuming tritons at rest. c) Differential cross-section $(d \sigma / d E)$ for production of neutrons vs. neutron energy ("energy spectrum") by mono-energetic protons (labels) incident on tritons at rest (solid lines) or tritons at $T=10 \mathrm{keV}$ (dashed lines, for clarity plotted only for $E_{p}=2,3$, and $4 \mathrm{MeV}$ ). The anisotropy in the $\mathrm{CM}$ frame causes the variation of the cross-section with energy. d) As in c), but evaluated for protons having energy distribution corresponding to temperatures 200-1000 keV (labels). Shown for tritons at rest and tritons at $10 \mathrm{keV}$ (dashed). The nearly complete overlap demonstrates that the thermal motion has insignificant effect. (b),c): cross-section and anisotropy data from [4]) 
kinematic anisotropy yields the differential reaction cross-sections in the lab frame shown in Fig. 2b. Forward scattering producing energetic neutrons is generally favoured and back-scatter largely suppressed particularly for $E_{p}<2 \mathrm{MeV}$.

If the high energy protons had isotropic velocity distribution in plasma the anisotropy of the pT neutron emission would average out. However, in ICRF-heated fusion plasmas, the high-energy protons are highly-anisotropic $\left(E_{\perp} \gg E_{\|}\right)$, hence, the neutron emission can be expected to be anisotropic as well. In particular, the high-energy neutrons will reflect the ion anisotropy. These neutrons will be emitted preferentially along the poloidal planes, nearly perpendicularly to the machine walls.

The energy spectrum of the pT neutrons can be found by combining the directiondependent energy and cross-section data. This has been done in Fig. 2c, which shows the reaction cross-section vs. neutron energy $(d \sigma / d E)$ for a few mono-energetic proton energies. Further, similar evaluation has been done for Maxwellian proton distributions of selected temperatures in Fig. 2d. The traces in Fig. 2d show the effective differential cross-section $(d \sigma / d E)$ for each proton in such distribution.

Thermal motion of tritons affects the reaction kinematics and could decrease the anisotropy of neutron emission. Relatively large effects are possible, in extreme cases: e. g. a $10 \mathrm{keV}$ triton can decrease the reaction threshold from $1019 \mathrm{keV}$ to $905 \mathrm{keV}$ for a 'head-on' collision. Numerical analysis of the thermal effects shows only a minor effect for forward-scattering well above threshold (i. e. high energy neutrons) but for backward-scattering, the thermal motion can randomise the direction and the energy of the scattered neutrons. The effects on neutron energy spectrum are shown in Fig. 2c and Fig. $2 \mathrm{~d}$ for $T=10 \mathrm{keV}$. Low energies are affected more, however, generally the spectrum is not much affected and averaging over proton energy distributions, the effect on the spectrum vanishes nearly completely (Fig. 2d). The main effect is the reduction of anisotropy of low-energy neutrons.

Overall, the anisotropy of pT reaction has impact on the shape of neutron spectrum. We have calculated the spectra in Fig. 2d also ignoring the CM anisotropy, and observed that neutron emission at low energies $(<0.5 \mathrm{MeV}$ ) is increased by up to $25 \%$ (for $T=1 \mathrm{MeV}$ ) while at $1-2 \mathrm{MeV}$ the emission is decreased by $\approx 30 \%$ when the anisotropy is taken into account. The effects are strongest with high proton temperatures.

The anisotropy could also affect the detection of the energetic, forward-scattered neutrons because they are ejected from plasma preferentially with small toroidal velocity component. Neutron diagnostics are calibrated assuming isotropic neutron emission, hence, pT neutrons could have a differing detection efficiency. If proton energy exceeds $3 \mathrm{MeV}$, some of the neutrons could, furthermore, be mistakenly measured as DD fusion neutrons. Computationally expensive simulations would be needed to evaluate this effect precisely. However, because these effects would only affect the very high-energy end of the overall neutron spectrum, i. e. small fraction of overall yield, these effects are considered as insignificant in this experiment. 


\subsection{Detection of the $p T$ reaction}

pT reaction can be detected by measuring the produced neutrons. The detection of the ${ }^{3} \mathrm{He}$ ions is not feasible: As the expected reaction rates are $\approx 10^{14} 1 / \mathrm{s}$ (Fig. 3 ), the plasma volume is $\approx 100 \mathrm{~m}^{3}$ and ion confinement time is $\approx 1 \mathrm{~s}$, one would expect a ${ }^{3}$ He density of $\approx 10^{12} 1 / \mathrm{m}^{3}$. This would be a factor $10^{7}$ below electron density and practically impossible to detect. Furthermore, ${ }^{3} \mathrm{He}$ is also produced in the DD reactions.

Measuring neutrons from pT reaction is challenging in fusion environment because the neutrons produced by the pT reaction have a broad, low-energy spectrum which will overlap the slowing-down neutrons from fusion reactions. Furthermore, the spectrum also depends strongly on the fast ion distribution (Fig. 2d). Ideally, if the contribution from the scattered neutrons could be eliminated, one would have one neutron spectrometer for the entire neutron spectrum and it would be straightforward to separate the broad-band pT neutrons outside the narrow-band emission from DD $(2.4 \mathrm{MeV})$ and DT $(14 \mathrm{MeV})$ fusion reactions. However, scattering of any fast neutrons in structural materials produces a wide energy spectrum, and the pT yield cannot be measured directly but it must be obtained by subtracting the contributions of the DD and DT fusion neutrons from the total spectrum.

The JET neutron spectrometers are set for measurements of the DD and DT neutrons, hence they are not ideal for the detection of the pT reaction. A scheme making use of several diagnostics simultaneously was developed. The excess neutron rate $R_{\text {exc }}$ is determined by subtracting from the total neutron rate $R_{n}$ the calibrated contributions from DD $\left(R_{\mathrm{DD}}\right)$ and $\mathrm{DT}\left(R_{\mathrm{DT}}\right)$ neutrons:

$$
R_{\mathrm{exc}}=R_{n}-\left(R_{\mathrm{DD}}+R_{\mathrm{DT}}\right) \text {. }
$$

$R_{n}$ is measured by fission chamber detectors situated at the iron limbs of JET[7], $R_{\mathrm{DD}}$ is measured by the JET neutron cameras[8], and $R_{\mathrm{DT}}$ by silicon diode detectors[9]. The term rate is used for time-dependent quantities. Analogously, for time-integrated neutron yields, $Y_{\mathrm{exc}}=Y_{n}-\left(Y_{\mathrm{DD}}+Y_{\mathrm{DT}}\right)$.

In addition to the $\mathrm{pT}$ reaction, other processes may produce extra neutrons in the plasma, especially if the proton tail temperature is extremely high. Nuclear reactions with plasma impurities, like ${ }^{9} \mathrm{Be}(p, n){ }^{9} \mathrm{~B}$ with reaction threshold of $2.1 \mathrm{MeV}$ become then possible. Energetic deuterons, if present, can also induce nuclear reactions with impurities or 'beam-target' type DD fusion reactions which produce neutrons with energy substantially differing from $2.4 \mathrm{MeV}$ due to kinematics (e.g. DD neutrons produced by $250 \mathrm{keV}$ deuterons have energy spread 2.0-3.1 MeV). Some of such neutrons may fall out of the energy window of the DD spectrometer and will not be accounted for in the DD neutron rate.

To assess the $\mathrm{pT}$ rate $R_{\mathrm{pT}}$, the effect from the other mechanisms needs to be considered. If they can be shown to be weak (e.g. due to the absence of very hot ion tail), the excess neutron rate can be attributed to pT reaction. Similarly, if the other processes are kept constant and the pT rate is varied (e.g. by varying tritium density), change in excess neutron rate can be attributed to the $\mathrm{pT}$ reaction. Furthermore, the 
absolute calibration of the pT yield is affected by the neutron spectrum: the low energy pT neutrons are more likely lost while traversing the JET machine structure than the DD and DT fusion neutrons. Due to the high computational cost of accurate calculation this has not been modelled in detail. It is most significant for low ion temperatures, and for relative comparisons with small differences in ion temperature this effect can be neglected.

Despite the difficulties, we believe that through careful analysis of the problems, good and meaningful results of $\mathrm{pT}$ neutron rate can be obtained. In this paper we have applied a conservative approach to analysing the different sources of error, and we limit the presentation to time- integrated or low time-resolution data, obtained predominantly during phases where $R_{\mathrm{DT}}$ is similar to or smaller than $R_{\mathrm{DD}}$, because high $R_{\mathrm{DT}}$ disturbs the $R_{\mathrm{DD}}$ measurement. In the companion paper [13], to extract the maximum amount of data with the highest possible time resolution, a different approach has been applied for eliminating sources of error from the experimental data.

\section{Modelling}

We have modelled the neutron rate and yield from the pT reactions using a generic but rather simple model as well as by carrying out dedicated simulations with PION code to optimise the experimental conditions for maximum yield. Because the reaction threshold is above $1 \mathrm{MeV}$, it is clear that very hot proton distributions are needed to produce appreciable pT neutron yield.

\subsection{Theoretical model}

pT reactions take place when highly energetic protons (with density $n_{p}(\mathbf{r}$ ) and distribution function $f(\mathbf{r}, E), \mathbf{r}$ is position vector) collide with tritons in the background plasma (density $n_{t}(\mathbf{r})$, temperature $T_{t}$ ). Because protons are far more energetic than tritons, $T_{t}$ is ignored and tritons are treated as immobile in the following. Local pT reaction rate $r_{\mathrm{pT}}(\mathbf{r})$ can be calculated by folding local fast proton flux $n_{p}(\mathbf{r}) f(\mathbf{r}, E) v(E)$, where $v$ is proton velocity, with the macroscopic reaction cross-section $n_{t}(\mathbf{r}) \sigma_{\mathrm{pT}}(E)$ :

$$
r_{\mathrm{pT}}(\mathbf{r})=\int_{0}^{\infty} n_{p}(\mathbf{r}) f(\mathbf{r}, E) v(E) \times n_{t}(\mathbf{r}) \sigma_{\mathrm{pT}}(E) d E
$$

The total pT reaction rate $R_{\mathrm{pT}}$ can then be found by integrating $r_{\mathrm{pT}}(\mathbf{r})$ over plasma volume $V$

$$
R_{\mathrm{pT}}=\int_{V} n_{p}(\mathbf{r}) n_{t}(\mathbf{r}) \int_{0}^{\infty} f(\mathbf{r}, E) v(E) \sigma_{\mathrm{pT}}(E) d E d \mathbf{r} .
$$

This integral is accurate, and can be evaluated numerically if the distribution function and profiles are known. However, it can be simplified if $f(\mathbf{r}, E)$ can be assumed to be of 2D-Maxwellian form $f_{T}(E)=1 / T \exp (-E / T)$ with local fast ion temperature $T(\mathbf{r})$. See Appendix for the justification of this form for highly anisotropic proton distributions. If 
local fast proton energy density is $w(\mathbf{r})$, proton density is then $n(\mathbf{r})=w(\mathbf{r}) / T(\mathbf{r})$. With these simplifications, the reaction rate can be expressed as

$$
R_{\mathrm{pT}}=\int_{V} n_{t}(\mathbf{r}) w(\mathbf{r}) / T(\mathbf{r}) \int_{0}^{\infty} f_{T}(\mathbf{r}, E) v(E) \sigma_{\mathrm{pT}}(E) d E d \mathbf{r} .
$$

As the integral over energy depends only on temperature it can evaluated independently (see Fig. 3) defining

$$
I_{\mathrm{pT}}(T) \equiv \frac{1}{T} \int_{0}^{\infty} f_{T}(E) v(E) \sigma_{\mathrm{pT}}(E) d E
$$

and the reaction rate becomes

$$
R_{\mathrm{pT}}=\int_{V} n_{t}(\mathbf{r}) w(\mathbf{r}) I_{\mathrm{pT}}(T(\mathbf{r})) d \mathbf{r} .
$$

This derivation is generic (within the assumptions made), i. e. by substituting appropriate microscopic cross-section for $\sigma_{\mathrm{pT}}$ and density for $n_{t}$, the equivalents of $I_{\mathrm{pT}}$ and $R_{\mathrm{pT}}$ can be evaluated for other nuclear reactions as well.

If the very fast ions are well-localised in the core of the plasma where the spatial dependence of $n_{t}(\mathbf{r})$ is weak, it can be replaced by a constant $n_{t}$ evaluated in the region of high fast ion density. Furthermore, if the tail of the entire fast proton population (at energies relevant for $\mathrm{pT}$ reaction) can be characterised adequately by a single temperature, all spatial dependence of the integral vanishes, and "simple model" for the $\mathrm{pT}$ reaction rate is obtained as

$$
R_{\mathrm{pT}}=n_{t} W_{\text {fast }} I_{\mathrm{pT}}(T),
$$

where $W_{\text {fast }}$ is total energy in fast ions. It should be noted that this $W_{\text {fast }}$ needs to be evaluated to match the actual proton tail above $E_{\text {th,lab }}$.

The simple model can be refined if the spatial temperature profile $T(r)$ around the peak at $r=r_{0}$ can be modelled by a parabolic form $T(r)=T_{0}\left(1-\left(\left(r-r_{0}\right) / b\right)^{2}\right)$ with half-width $b$ and peak temperature $T_{0}$. Integrating from $r_{0}-b$ to $r_{0}+b$, one obtains a spatially averaged distribution function $F_{\text {par }}(E)$ of the form

$$
F_{\text {par }}(E)=\frac{1}{2 T_{0}} \mathrm{~K}_{0}\left(\frac{E}{2 T_{0}}\right) \exp \left(-\frac{E}{2 T_{0}}\right)
$$

where $\mathrm{K}_{0}$ is the Bessel $\mathrm{K}$ function. The average particle energy in this distribution is $<E>=2 T_{0} / 3$. The derivation is carried out for plasma slab but it is valid for other plasma shapes when flux surfaces have the same shape and a common centre point for $r_{0}-b<r<r_{0}+b$. It is not valid near the plasma centre, $r_{0}<b$, because symmetry around $r=r_{0}$ is broken.

At high energies $\left(E>T_{0}\right)$, the asymptotic form $\mathrm{K}_{0}(x) \approx \sqrt{\pi / 2 x} \exp (-x)$ can be substituted, resulting in the form

$$
F_{\mathrm{par}}(E)=\sqrt{\frac{\pi}{4 T_{0} E}} \exp \left(-\frac{E}{T_{0}}\right) .
$$


At high energies relevant to $\mathrm{pT}$ fusion this can be approximated by single Maxwellian distribution but with effective temperature less than $T_{0}$. It is also possible to define $I_{\mathrm{pT}, \mathrm{par}}\left(T_{0}\right)$, the exact equivalent to Eq. 5:

$$
I_{\mathrm{pT}, \mathrm{par}}\left(T_{0}\right) \equiv \frac{3}{2 T_{0}} \int_{0}^{\infty} F_{\mathrm{par}}(E) v(E) \sigma_{\mathrm{pT}}(E) d E .
$$

This is also plotted in Figure 3. It is seen that the pT rate is less than for singleMaxwellian model. This is because the average ion temperature is lower for the parabolic distribution than for a single temperature distribution.

The table in Fig. 3 shows the expected pT neutron rate for $W_{\text {fast }}=1 \mathrm{MJ}$ and $n_{t}=10^{17} \mathrm{~m}^{-3}$ (broadly typical for JET TTE plasma). As the rate is proportional to $n_{t}$, about 1000 times higher rate could be expected in fusion plasma conditions $n_{t}=10^{20} \mathrm{~m}^{-3}$. It is seen from Eq. 7 that the pT neutron rate depends linearly both on $n_{t}$ and $W_{\text {fast }}$ when $T_{\text {fast }}$ is kept constant. Contrary to this, $I_{\mathrm{pT}}(T)$ has a highly nonlinear and strong dependence on $T_{\text {fast }}$. The increase with temperature is mainly due to the increase in number of protons exceeding the threshold. Another contribution comes from the fact that, with hotter distributions, there are more protons close to the peak of the cross-section (Fig. 1). Finally, the decrease with $T_{\text {fast }}>1 \mathrm{MeV}$ is caused because $N_{p}$ decreases due to the increasing average proton energy.

In principle, the strong dependence on fast ion temperature suggests that $\mathrm{pT}$ reaction could have diagnostic applications. If $R_{\mathrm{pT}}, n_{t}$ and $W_{\text {fast }}$ can be determined by other diagnostics $T_{\text {fast }}$ could be determined from the reactivity $I_{\mathrm{pT}}$. $R_{\mathrm{pT}}$ could be measured by measuring the pT neutron emission, $n_{t}$ possibly by optical means, neutral particle fluxes or estimated from DD and DT fusion neutron rates and $W_{\text {fast }}$ could be determined by magnetic or neutral particle measurements. If $T_{\text {fast }}$ can also be determined by other means (like neutral particle measurements) pT reaction could be used for measuring $n_{t}$. However, with the practical diagnostic limitations, creating an useful diagnostic appears doubtful. A more advanced diagnostic could be contemplated if there are advances in neutron spectroscopy: as the pT neutron spectrum depends strongly on ion spectrum, it might be possible to deduce information about the fast ion distribution from the neutron spectrum.

2.1.1. How good is the simple model? In real plasmas, the total fast ion distribution does not have the Maxwellian form assumed in the simple model because fast ion temperature is not spatially constant, nor are density profiles flat. However, often the fast ions are well localised, and for $\mathrm{pT}$ reaction, only the hot tail above $1 \mathrm{MeV}$ matters. To estimate the errors of the simple model, we have evaluated the total fast ion spectrum using a more realistic description assuming a locally Maxwellian distribution, parabolic density profiles and Gaussian fast ion temperature profiles. This is equivalent to comparing the simple model (Eq. 7) to Eq. 6 which takes the spatial dependencies into account.

Figure 4 shows the results of the comparison, assuming a Gaussian half-width of $0.2 a$ and profile centered on plasma axis. It is seen that the simple Maxwellian fit 


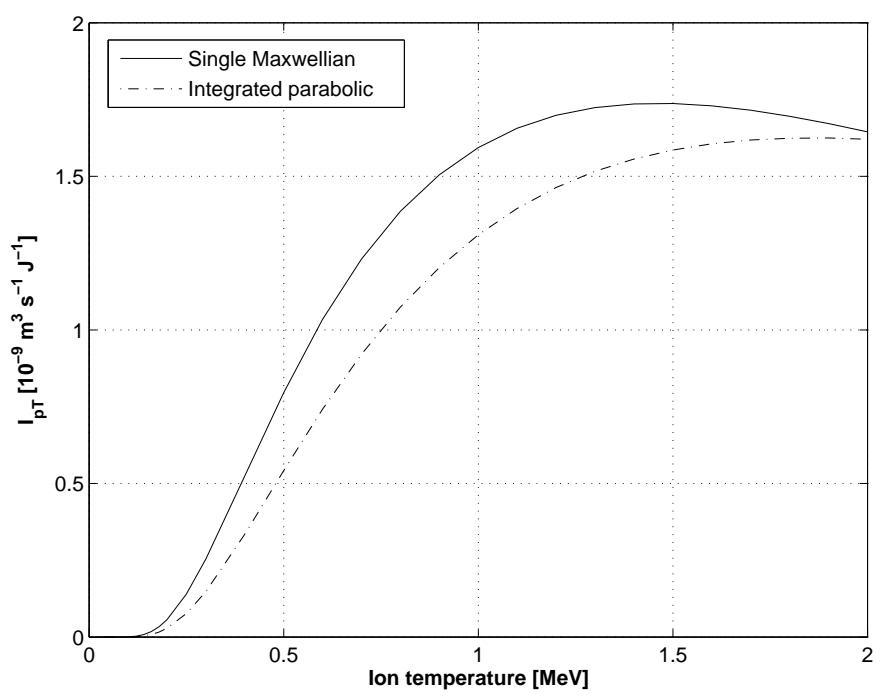

\begin{tabular}{|c|c|c|}
\hline $\begin{array}{c}T \\
{[\mathrm{keV}]}\end{array}$ & $\begin{array}{c}R_{\mathrm{pT}} \\
{[1 / \mathrm{s}]}\end{array}$ & $\begin{array}{c}R_{\mathrm{pT}, \mathrm{par}} \\
{[1 / \mathrm{s}]}\end{array}$ \\
\hline 100 & $5.0 \times 10^{10}$ & $1.9 \times 10^{10}$ \\
200 & $5.6 \times 10^{12}$ & $2.8 \times 10^{12}$ \\
300 & $2.5 \times 10^{13}$ & $1.5 \times 10^{13}$ \\
400 & $5.3 \times 10^{13}$ & $3.3 \times 10^{13}$ \\
500 & $8.0 \times 10^{13}$ & $5.4 \times 10^{13}$ \\
600 & $1.0 \times 10^{14}$ & $7.4 \times 10^{13}$ \\
800 & $1.4 \times 10^{14}$ & $1.1 \times 10^{14}$ \\
1000 & $1.6 \times 10^{14}$ & $1.3 \times 10^{14}$ \\
2000 & $1.7 \times 10^{14}$ & $1.6 \times 10^{14}$ \\
\hline
\end{tabular}

Figure 3. The overlap integral $I_{\mathrm{pT}}(T)$ for single-temperature $T$ Maxwellian distribution (solid) and $I_{\mathrm{pT} \text {,par }}\left(T_{0}\right)$ for integrated parabolic temperature distribution with peak temperature $T_{0}$ (dashed). The table on right shows calculated $\mathrm{p} T$ reaction rates for $W_{\text {fast }}=1 \mathrm{MJ}$ and $n_{t}=10^{17} \mathrm{~m}^{-3}$ for the single-temperature and parabolic models.

a)

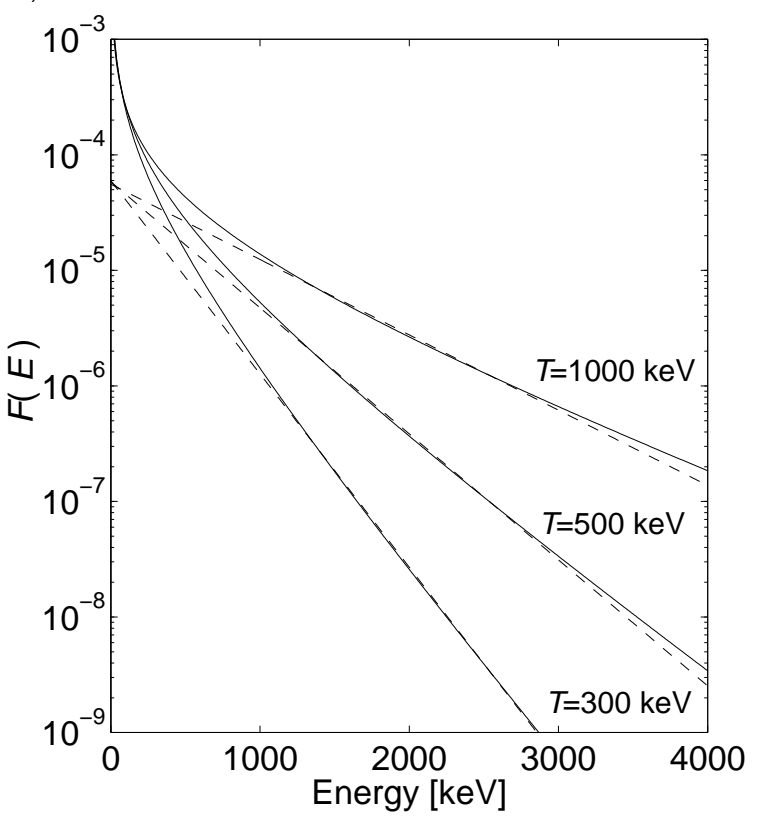

b)

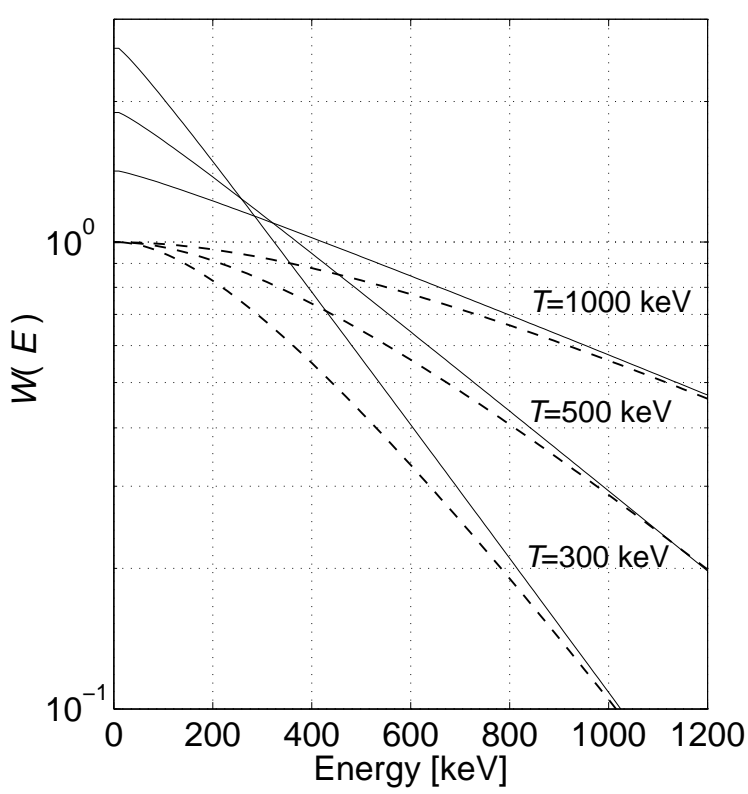

Figure 4. Comparison of the simple model (dashed) and more realistic description of the fast ion distribution (solid) (see text). (a) Fast ion distribution for $T_{\text {fast }}=300$, 500 , and $1000 \mathrm{keV}$. Straight lines show best exponential fit in the interval $1-3 \mathrm{MeV}$. Best fit temperatures for simple model are 260, 400, and $660 \mathrm{keV}$ (b) Fast ion energy content $W\left(E_{0}\right)$ as function of the low-energy cut-off $E_{0}$, normalised so that $\mathrm{W}(0)=1$ for Maxwellian fit. 
describes very well (errors $<5 \%$ ) the more realistic distribution at energies relevant for the pT reaction $(>1 \mathrm{MeV})$. The fitted temperature, $T_{\text {fit }}$ however, is lower than the peak local temperature. As substantial fraction of the total energy content comes from off-axis plasma regions the error in $W_{\text {fast }}$ becomes substantial and must be corrected for to obtain realistic values for the calculation of the $\mathrm{pT}$ rate. This correction depends strongly on the low-energy cutoff for $W_{\text {fast }}$ measurement, and the correction is largest for fast ion distributions having peak temperature at plasma centre.

The parabolic model is more accurate than single-Maxwellian model as it properly takes the lower energy off-peak ion population into account. However, the derivation is not correct for centre-peaked distributions. Comparing pT reaction rates (in Fig. 3) for parabolic model at peak temperature $\left(I_{\mathrm{pT} \text {,par }}\left(T_{\text {fast }}\right)\right)$ to Maxwellian model at best fit temperature $\left(I_{\mathrm{pT}}\left(T_{\text {fit }}\right)\right)$ shows nevertheless a fairly good fit even in the centre-peaked case.

If the fast ion distribution and tritium concentration are known accurately it is a matter of straightforward volume integration of the spatially dependent reaction rate to calculate the pT yield accurately. This can be done, e.g., in PION simulations. The simple models serve to illustrate the most important physical dependencies of the pT reaction yield for rough estimations.

\subsection{PION simulations}

In order to find the parameter regime where maximal $\mathrm{pT}$ yield with respect to the DD and DT neutron yield is obtained, a series of simulations were carried out using the PION code [10] prior to the experiment. As a reference discharge, a recent $3.4 \mathrm{~T} / 1.8 \mathrm{MA}$ discharge (JET discharge 57298) with central electron density $n_{e}(0) \approx 2.5 \times 10^{19} \mathrm{~m}^{-3}$ and temperature $T_{e}(0) \approx 5.5 \mathrm{keV}$ using about $7 \mathrm{MW}$ of hydrogen minority heating with a central resonance was selected. The presence of high-energy proton tail in this discharge had been experimentally confirmed by gamma ray emission from reactions of fast protons with thermal deuterons and ${ }^{9}$ Be impurity ions, by high-energy NPA measurements and observation of fast-ion driven Alfven eigenmodes. However, gamma and neutron data also indicated a population of fast deuterons, driven at their second harmonic ion cyclotron resonance which coincides with the fundamental hydrogen resonance. These fast deuterons were unwanted in the pT experiment since they would have enhanced the DD and DT neutron yield with respect to the pT yield and therefore make pT reaction more difficult to detect. The main parameter that was varied in the simulations was the hydrogen concentration as it plays an important role in the power partitioning between the minority proton and majority deuteron populations, see e.g. Ref. [11].

Figure 5 a shows the DD, DT and pT reaction rate calculated by PION using the parameters of reference discharge 57298 and assuming a tritium to deuterium density ratio of $1 \%$ and an ion temperature equal to half of the electron temperature. It should be noted that PION simulations include a more detailled modelling of the fast ion tail than what is taken into account with the simple analytic model. As the hydrogen 
a)

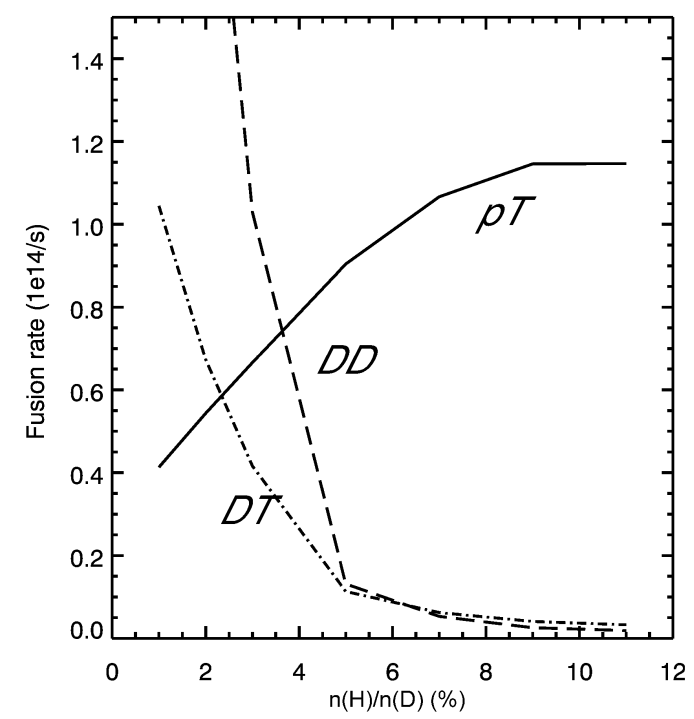

c)

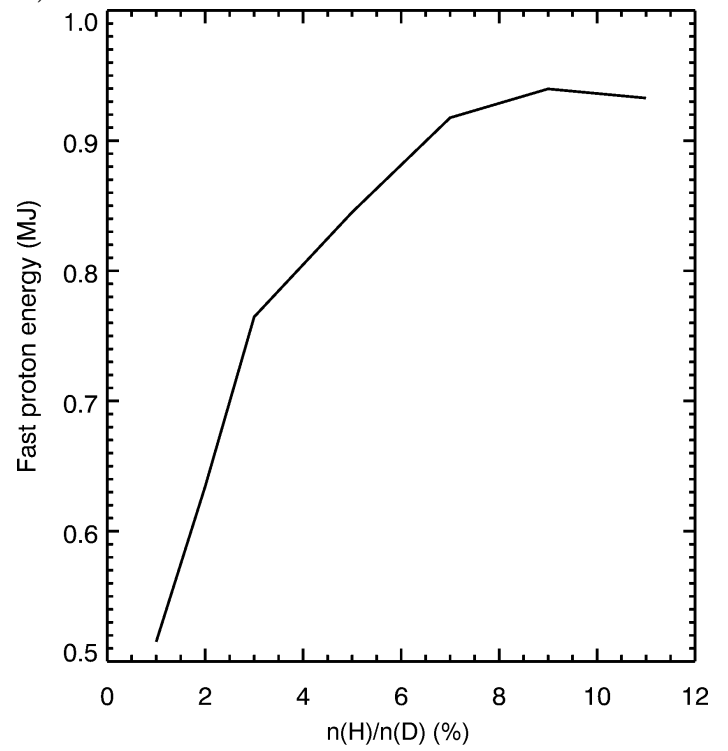

b)

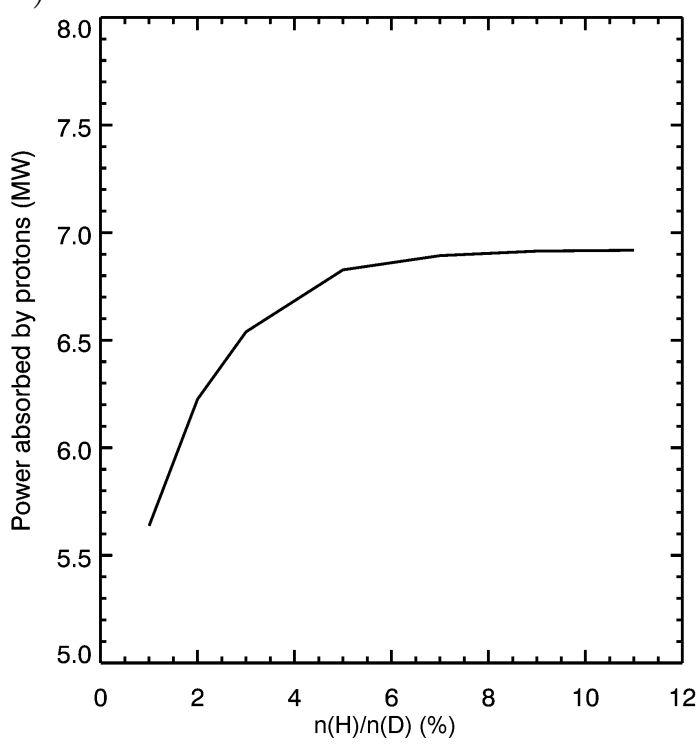

d)

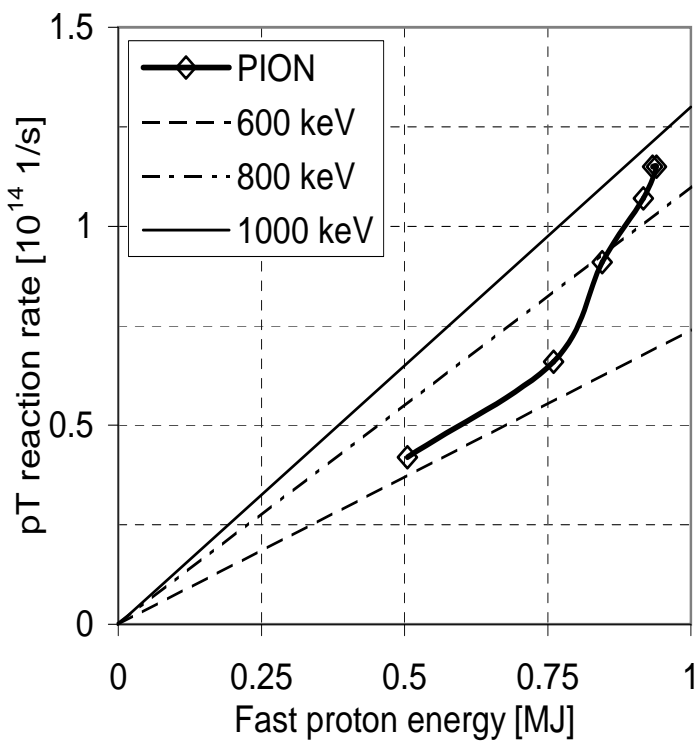

Figure 5. Results from PION simulations of the reference pulse 57298 as function of hydrogen concentration (see text) a) reaction rates for DT, DD and pT with $1 \%$ of $\mathrm{T}$ in plasma, b) RF power absorbed by protons with $7 \mathrm{MW}$ input, c) total energy of the fast protons, and d) pT reaction rate vs. proton energy content, with expected $\mathrm{pT}$ reaction rate at different peak temperatures, using the parabolic model. 
concentration is increased, the power absorbed by deuterium decreases and the DD and DT fusion rates decrease to the thermal level. At the same time, power absorbed by hydrogen increases (Fig. 5b), increasing the pT reaction rate and the fast proton energy content (Fig. 5c). The absolute level of the calculated pT and DT rates relative to the DD rate depends on the assumed tritium concentration as well as on the assumed ion temperature. Based on the simulation results, a hydrogen concentration in the range of $5-10 \%$ was selected as a target for the experiment. Furthermore, $+90^{\circ}$ phasing between the currents in the four straps of the JET ICRF antennas was chosen due to its capability to enhance the high-energy part of the ICRF driven fast proton population due to wave-induced inward radial pinch of the resonant ions [12].

\section{Experiment}

The pT reaction experiment was carried out during the JET Trace Tritium Experiment (TTE). Small amounts of tritium were introduced into deuterium majority plasma either by gas puffing or by neutral beams, though only puffing was used with pT experiment. Typical tritium density was on the order of $10^{17} \mathrm{~m}^{-3}$ (i. e. less than $1 \%$ of electron density), though momentarily after puffing the density could be much higher. Applying the simple model, Eq. 7, one would expect pT reaction rate of $0.8-1.6 \times 10^{14}$ for $W_{\text {fast }}=1 \mathrm{MJ}$ and $T_{\text {fast }}=500-1000 \mathrm{keV}$. This is consistent with the predictions of the PION simulations (Fig. 5).

Because a high DT neutron rate disturbs the measurement of the DD neutron rate (the neutron camera used for DD measurement is also sensitive to DT), it was desirable to keep the DT fusion rate low while maximising the $\mathrm{pT}$ rate. With low tritium densities, both DD an pT rates are proportional to density. Hence, variations in tritium density affect the detectability of $\mathrm{pT}$ reaction only indirectly by affecting the accuracy of the DD neutron rate measurement. Both DD and DT fusion neutrons are unwanted background to the pT reaction. To keep their production low, it was desireable to keep the bulk ion temperature low and avoid the formation of suprathermal deuteron populations.

A typical pulse in this experiment is shown in Figure 6. The nominal toroidal field was $3.4 \mathrm{~T}$ and plasma current 1.8 MA. During the flat-top phase, the maximum available ICRF heating power was applied. Two heating schemes were used: monochromatic $(51 \mathrm{MHz})$ with resonance at major radius $3.0 \mathrm{~m}$ and polychromatic (two frequencies: $47 \& 51 \mathrm{MHz}$ ) with resonances at major radii 3.0-3.3 m. A varying amount of tritium was optionally puffed into plasma just before reaching the maximum $R F$ power. A short (200 ms) pulse of deuterium neutral beam heating ("NBI blip") was applied to the plasma late in the pulse for charge-exchange (CX) measurements.

Table 1 shows the main data for the seven plasma pulses in the pT reaction experiment. The two first pulses (61254 and 61256) were repeats of the reference pulse without addition of tritium. The next two pulses were repeats of 61256 but with increasing tritium puffing (3.0 and $5.1 \mathrm{mg}$ ). The following two pulses (61259 and 61260) are like 61257 but with polychromatic RF heating. The last pulse, 61261, is repeat of 


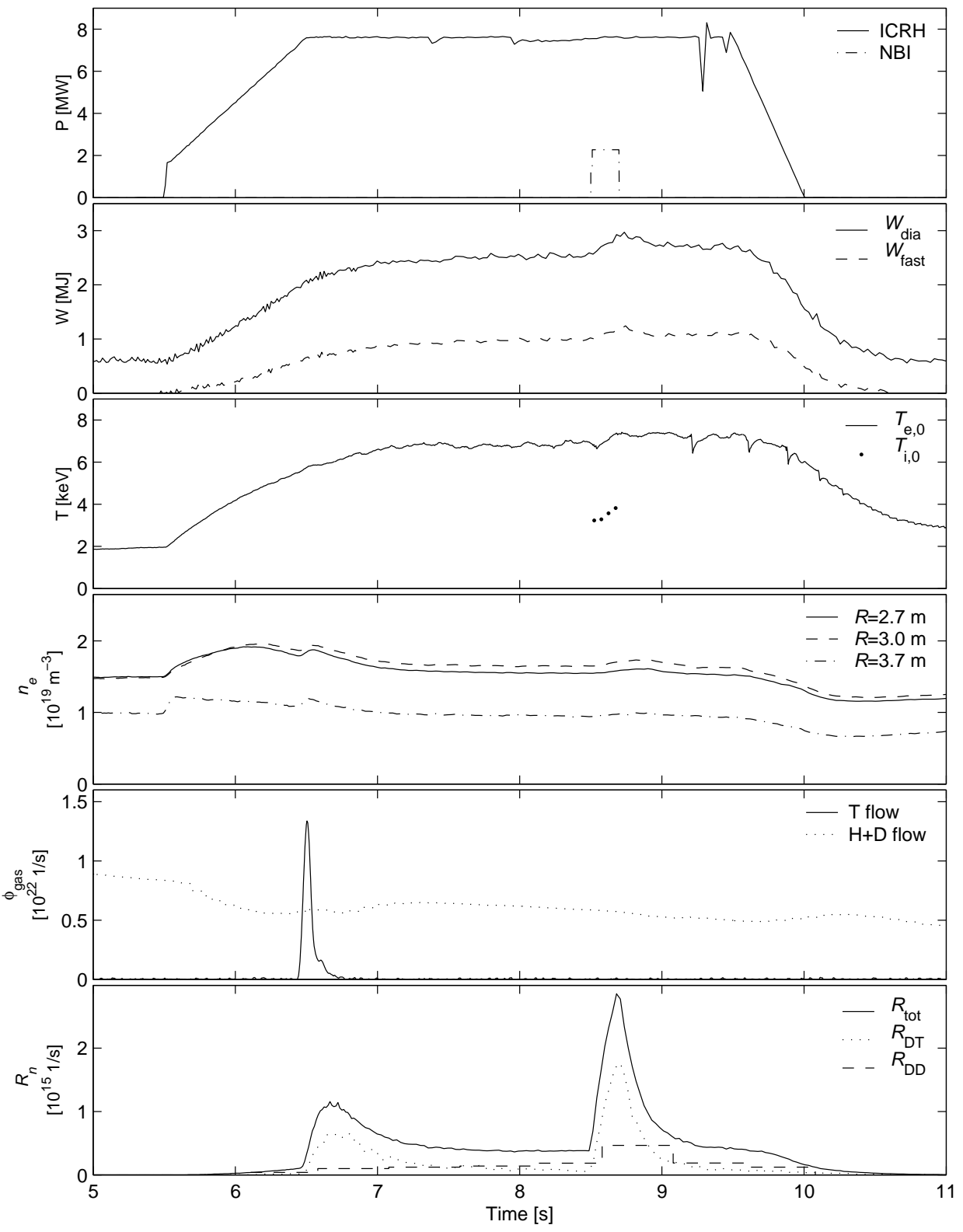

Figure 6. The main plasma parameters in a typical pulse (61258). ICRH and NBI are the RF and neutral beam heating power, $W_{\text {dia }}$ and $W_{\text {fast }}$ are the diamagnetic energy and the energy in fast ions $\left(W_{\mathrm{fast}}=2 / 3\left(W_{\mathrm{dia}}-W_{\mathrm{blk}}\right), W_{\mathrm{blk}}\right.$ is the total energy in thermal electrons and ions), $T_{e, 0}$ and $T_{i, 0}$ are thet electron and ion on-axis temperatures from LIDAR and charge-exchange spectroscopy, $n_{e}$ are the line-averaged electron densities from laser interferometry, $\Phi_{\text {gas }}$ are the gas puffing rates, and $R_{n}$ are the neutron rates. 
Table 1. Main data of the plasma discharges in the pT reaction experiment. Neutron data is time-integrated up to NBI blip (8.5 s).

\begin{tabular}{|c|c|c|c|c|c|c|c|c|c|c|}
\hline Pulse & $\begin{array}{c}\text { RF } \\
{[\mathrm{MHz}]}\end{array}$ & $\begin{array}{c}\text { Power } \\
{[\mathrm{MW}]}\end{array}$ & $\begin{array}{c}\text { H conc } \\
\%\end{array}$ & $\begin{array}{c}\text { T puff } \\
{[\mathrm{mg}]}\end{array}$ & $\begin{array}{c}T_{\mathrm{NPA}} \\
{[\mathrm{keV}]}\end{array}$ & $\begin{array}{c}W_{\text {fast }} \\
{[\mathrm{MJ}]}\end{array}$ & $\begin{array}{c}Y_{n} \\
\times 10^{14}\end{array}$ & $\begin{array}{c}Y_{\mathrm{DT}} \\
\times 10^{14}\end{array}$ & $\begin{array}{c}Y_{\mathrm{DD}} \\
\times 10^{14}\end{array}$ & $\begin{array}{c}Y_{\text {exc }} \\
\times 10^{14}\end{array}$ \\
\hline 61254 & 51 & $6.6-7.2$ & 2.9 & 0.0 & 440 & 0.54 & 3.2 & 0.08 & 2.2 & 1.0 \\
61256 & 51 & $6.3-7.2$ & 5.2 & 0.0 & 450 & 0.59 & 3.4 & 0.08 & 2.2 & 1.2 \\
61257 & 51 & $7.1-7.4$ & 6.6 & 3.0 & 480 & 0.60 & 7.7 & 2.7 & 2.9 & 2.1 \\
61258 & 51 & $7.4-7.6$ & 8.0 & 5.1 & 450 & 0.63 & 11 & 4.7 & 3.3 & 3.0 \\
61259 & $47 / 51$ & $4.6-5.6$ & 6.0 & 3.2 & 430 & 0.48 & 5.1 & 2.7 & 1.2 & 1.2 \\
61260 & $47 / 51$ & $4.3-6.3$ & 5.3 & 3.0 & 440 & 0.48 & 4.9 & 2.7 & 1.3 & 0.9 \\
61261 & 51 & $2.7-4.5$ & 6.1 & 5.1 & 280 & 0.11 & 3.8 & 2.8 & 0.3 & 0.7 \\
\hline
\end{tabular}

61258 but with a substantially lower RF power. The minor differences compared to [13], e.g., in neutron yield arise because of different methods used in the analysis. The $W_{\text {fast }}$ in Table 1 has been evaluated from diamagnetic measurements assuming linear dependency for pedestal during RF phase.

The achieved pulse comparisons are: (A) 61256-61258 — identical pulses with different tritium feeding (0, 3 and $5 \mathrm{mg}$ ), (B) 61257 vs. 61259 and 61260 monochromatic vs. polychromatic heating at the same level of tritium feeding (3 mg) and (C) 61258 vs. 61261 - similar pulses but with substantially different RF heating power. Similar RF power was applied to the plasma in all pulses, apart from pulse 61261.

\section{Results}

The time-integrated excess neutron yield was evaluated by integrating the neutron signals until the NBI blip, shown in Table 1. A substantial neutron excess is seen in all pulses. Similar calculations have been carried out for a number of other pulses, and the result as neutron excess fraction is shown in Fig. 7. It is seen that a substantial excess neutron fraction is only observed with the $\mathrm{pT}$ reaction pulses. The scatter of the other points around zero is likely to reflect the systematic inaccuracies in the neutron diagnostics.

The experimental pulses were planned to have as little MHD activity as possible. An analysis of magnetics signals shows that this goal was achieved repeatedly. Sawtooth crashes were present but their amplitude was low, particularly before the NBI blip. Fastion-driven Alfven modes were present in all pulses apart from the 61261 with low RF power. Fishbone instabilities were observed in 61257 and 61258 but only after the NBI blip. Hence, it appears that MHD activity has similar and small impact on the fast ions making the pulses a good basis for the study of $\mathrm{pT}$ reaction and pulse-to-pulse 


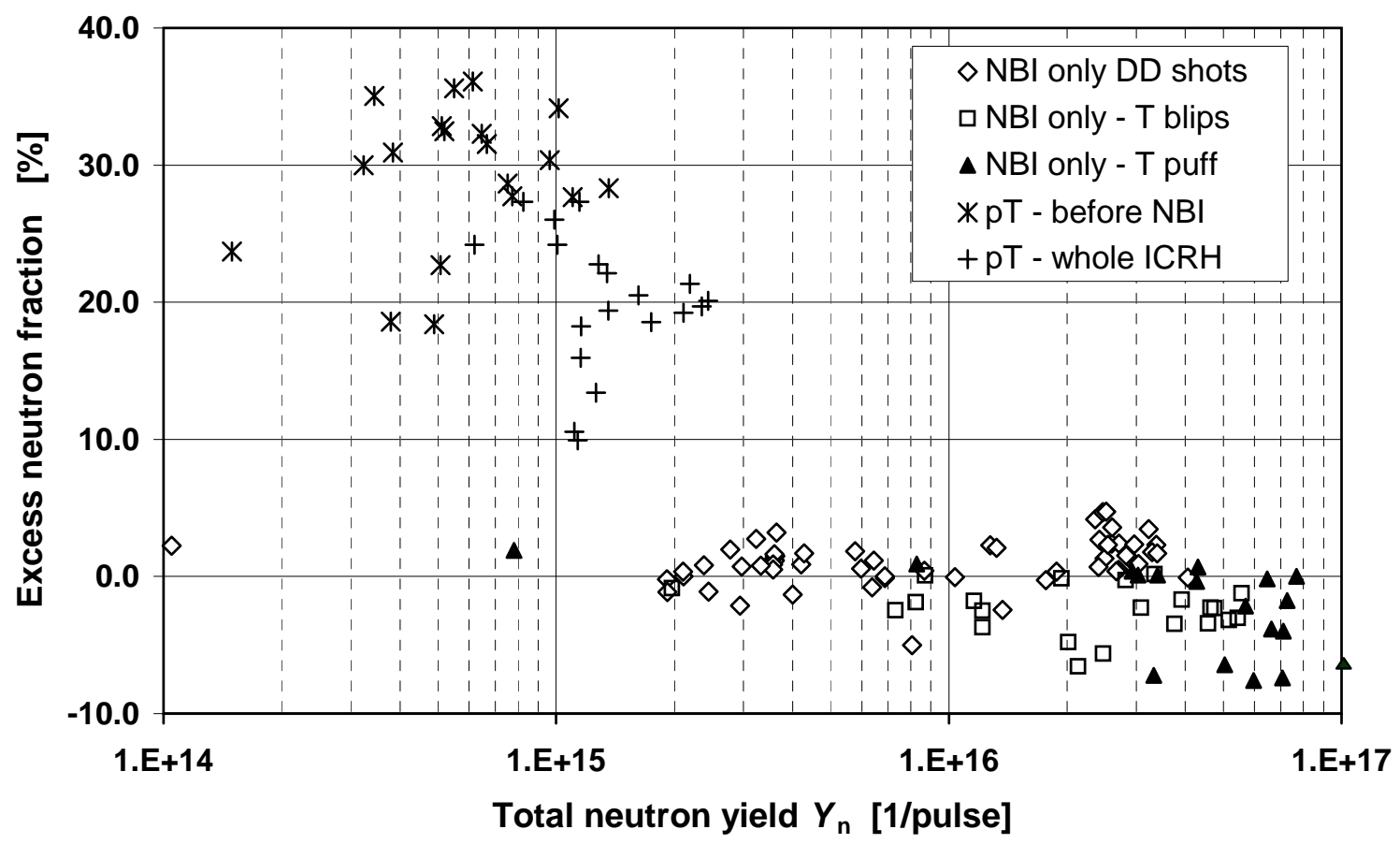

Figure 7. Production of 'excess' neutrons in a number of pulses in the TTE. The points indicate excess as percentage of total measured neutron production.

comparisons feasible. The only exception is pulse 61261 where more sawtooth activity and no Alfven modes were observed, and magnetic surfaces also differed slightly from the other pulses.

The presence of the fast particles in the plasma was established by neutral particle flux measurements and by gamma spectroscopy. Table 1 shows the average fast ion temperature (by NPA) between 6.5 and $8.5 \mathrm{~s}$ i. e. during highest RF heating but before the NBI blip. Apart from 61261, fairly similar temperatures close to $0.5 \mathrm{MeV}$ are observed, regardless of the heating scheme. The energy range of the NPA measurement was $290-1100 \mathrm{keV}$. At higher energies, fast ions were detected through gamma emission from ${ }^{12} \mathrm{C}\left(p, p^{\prime} \gamma\right){ }^{12} \mathrm{C}$ reaction with threshold of $5 \mathrm{MeV}$ and gamma energy $E_{\gamma}=4.44 \mathrm{MeV}$. In the high-power monochromatic pulses, strong gamma emission was observed as seen in Fig. 8. In addition to the emission from ${ }^{12} \mathrm{C}\left(p, p^{\prime} \gamma\right){ }^{12} \mathrm{C}, 3.09-\mathrm{MeV}$ gammas from the reaction ${ }^{12} \mathrm{C}(d, p \gamma){ }^{13} \mathrm{C}$ were observed. This implies that some parasitic deposition of ICRF power on the bulk deuterons occurred. In pulses with tritium puffing the 4.44-MeV signal is superimposed with background from ${ }^{12} \mathrm{C}\left(n, n^{\prime} \gamma\right){ }^{12} \mathrm{C}$ induced by the $14-\mathrm{MeV}$ neutrons (spikes at tritium puffing and NBI blip). In the polychromatic and low-power monochromatic cases (61259-61261) only the background correlated with DT neutron emission is present, suggesting very weak or non-existent proton population above $5 \mathrm{MeV}$.

Other, indirect indication of very high energy protons can be seen in the NPA signals when the RF power is switched off: in the monochromatic case, NPA flux is 
a)

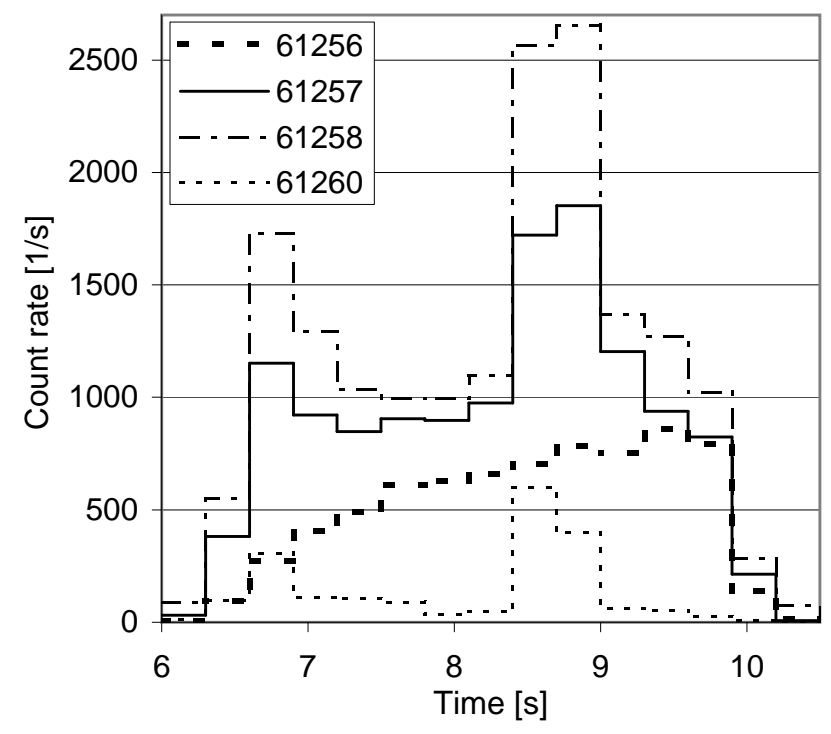

b)

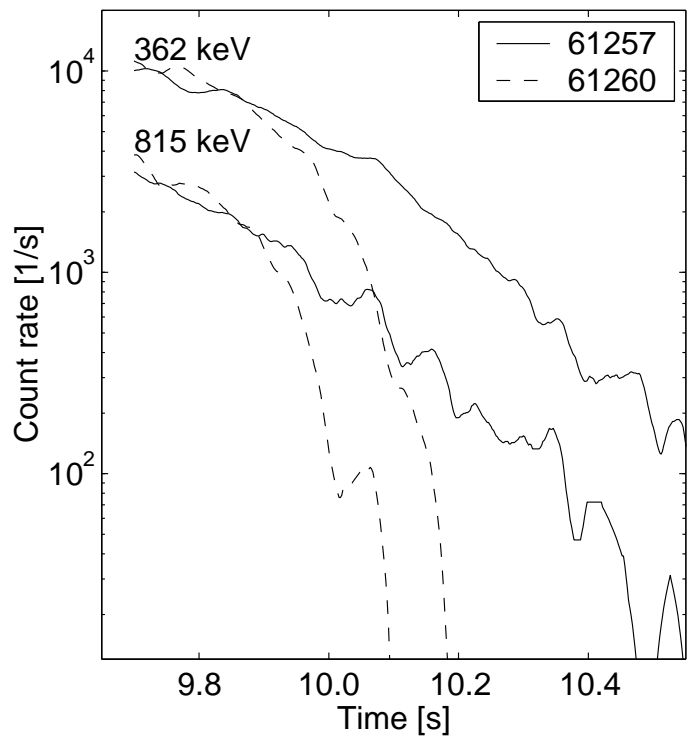

Figure 8. a) 4.44-MeV $\gamma$-ray emission from the reaction ${ }^{12} \mathrm{C}\left(p, p^{\prime} \gamma\right){ }^{12} \mathrm{C}$. b) NPA flux at the end of RF heating. RF power is ramped down linearly berween 9.5 and $10 \mathrm{~s}$.

present for $0.3-0.7 \mathrm{~s}$ after $\mathrm{RF}$ power has been reduced to zero while in polychromatic case the flux disappears in 0.0-0.2 s (Fig. 8b). This difference cannot fully be explained by greater (up to $2 \times$ ) ion stopping power in the polychromatic case, and is likely to arise from high energy ions slowing down into the NPA energy window. Assuming that no ion acceleration takes place when RF is switched off and integrating ion stopping power in plasma, it can be estimated that, e. g., ions seen at $455 \mathrm{keV}$ window at $10.5 \mathrm{~s}$ have had $2.2 \mathrm{MeV}$ energy at $10.0 \mathrm{~s}$.

Overall, the pulses have good conditions for producing $\mathrm{pT}$ reaction: they have low MHD activity, and multiple diagnostics indicate the presence of large, very hot proton population. Indeed, substantial neutron excess is also observed. The significance of the other possible mechanisms (like impurity-induced reactions or beam-type fusion by fast deuterons) needs to be considered, however. In the setup pulses 61254 and 61256 , a substantial neutron excess $\left(\approx 1 \times 10^{14} \mathrm{n}\right)$ was observed, but the absence of $\mathrm{T}$ puffing and the low DT neutron yield $(<3 \%$ of the yield with tritium puffing, see Table 1$)$ shows that the tritium concentration (arising from recycling at wall) is very low. The setup pulses were preceded by three pulses with no tritium puffing. Hence, the excess in the setup pulses can be seen as arising from the non-pT sources only, at rate of $0.4 \pm 0.1 \times 10^{14} \mathrm{n} / \mathrm{s}$.

pT reaction is observed in the following pulses with increasing tritium puffing, otherwise identical to 61256. The time-integrated neutron excess is seen to increase: excess was $1.2,2.1$, and $3.0 \times 10^{14} \mathrm{n}$ with tritium puffing of $0.0,3.0$ and $5.1 \mathrm{mg}$ in pulses 61256, 61257, and 61258, respectively. In time-resolved signals, a strong correlation is observed between excess and DT neutron rates as well as between excess rate and $R_{\mathrm{DT}} / R_{\mathrm{DD}}$ ratio (Fig. 10). The neutron excess which is correlated with $R_{\mathrm{DT}}$ is strong 
evidence of $\mathrm{pT}$ reaction taking place as both scale linearly with tritium density. As the best fit to data is extrapolated to $R_{\mathrm{DT}}=0$, the contribution from the non-pT sources is obtained, well matching the measured rate in the setup pulse 61256 .

The polychromatic pulses 61259 and 61260 have lower neutron excess than the monochromatic ones which is consistent with larger power deposition volume and consequently lower proton tail temperature. The lower tail temperature is clearly observed through gamma measurements with very weak, if any, signal, and also in rapid decrease of the NPA signal after RF switch-off. The NPA spectra, measured below $1100 \mathrm{keV}$, show only little reduction in ion temperatures compared to the monochromatic cases, however. As the non-pT excess production relies on having a strong, highly energetic tail, and such a tail is not observed it can be argued that the non-pT mechanisms have a negligible role in the polychromatic pulses, and the excess can be attributed to the pT reaction. The best fit to data in the Figure 10 crosses $R_{\mathrm{DT}}=0$ close to $R_{\mathrm{exc}}=0$, consistent with the absence of the non-pT mechanisms. The DD and DT neutron rates are of similar magnitude in these pulses which makes it difficult to evaluate the neutron excess accurately using standard techniques, and this increases the scatter in data. The last pulse, 61261, is affected even more by this, and extended analysis also involving help from modelling is needed to obtain excess data, as presented in the companion paper [13].

Figure 9 shows the temporal dependence of the neutron rates in selected pulses. In 61256, fairly constant neutron rates are observed apart from the increased neutron emission during the NBI blip. A nearly constant neutron excess is also seen. In 61257 and 61258 increasing amounts of tritium is puffed. Both the DT neutron rate and excess neutron rate increase after the puff and decay slowly afterwards as the tritium concentration in plasma first jumps up and then slowly decays. During the NBI blip, the DD and DT rates increase sharply due to beam-induced fusion reactions, however, the excess is affected only a little. This is consistent with that the neutron excess is driven by very fast ions which are not affected by the NBI blip. Similar behaviour is also seen with pulse 61260, however, the excess is substantially lower and there is more scatter in the signal. This could result from inaccuracies in the DD neutron rate measurement with increasing DT neutron fraction ( $\approx 50 \%$ of total). In 61261 (not shown, DT fraction about $75 \%$ ), the scatter in excess data is so large that data is directly of little use.

If the fast ion population and bulk plasma temperature is more or less constant and repeatable from pulse to pulse one expects $R_{\mathrm{pT}}, R_{\mathrm{DT}}$ and $R_{\mathrm{DT}} / R_{\mathrm{DD}}$ ratio to scale linearly with tritium density. In figure 10 the calculated neutron excess rate is plotted against DT neutron rate and DT/DD rate ratio. It is seen that the points from the monochromatic pulses 61256-8 follow a straight line quite well, apart from a few outlying points. The point during the NBI blip is not expected to be on the line due to beaminduced DT and DD fusion, and the points during T puffing have very high DT neutron fraction affecting the DD data quality momentarily. The polychromatic pulses show a smaller neutron excess compared to DT yield, as expected for pulses with weaker hot ion tail. 

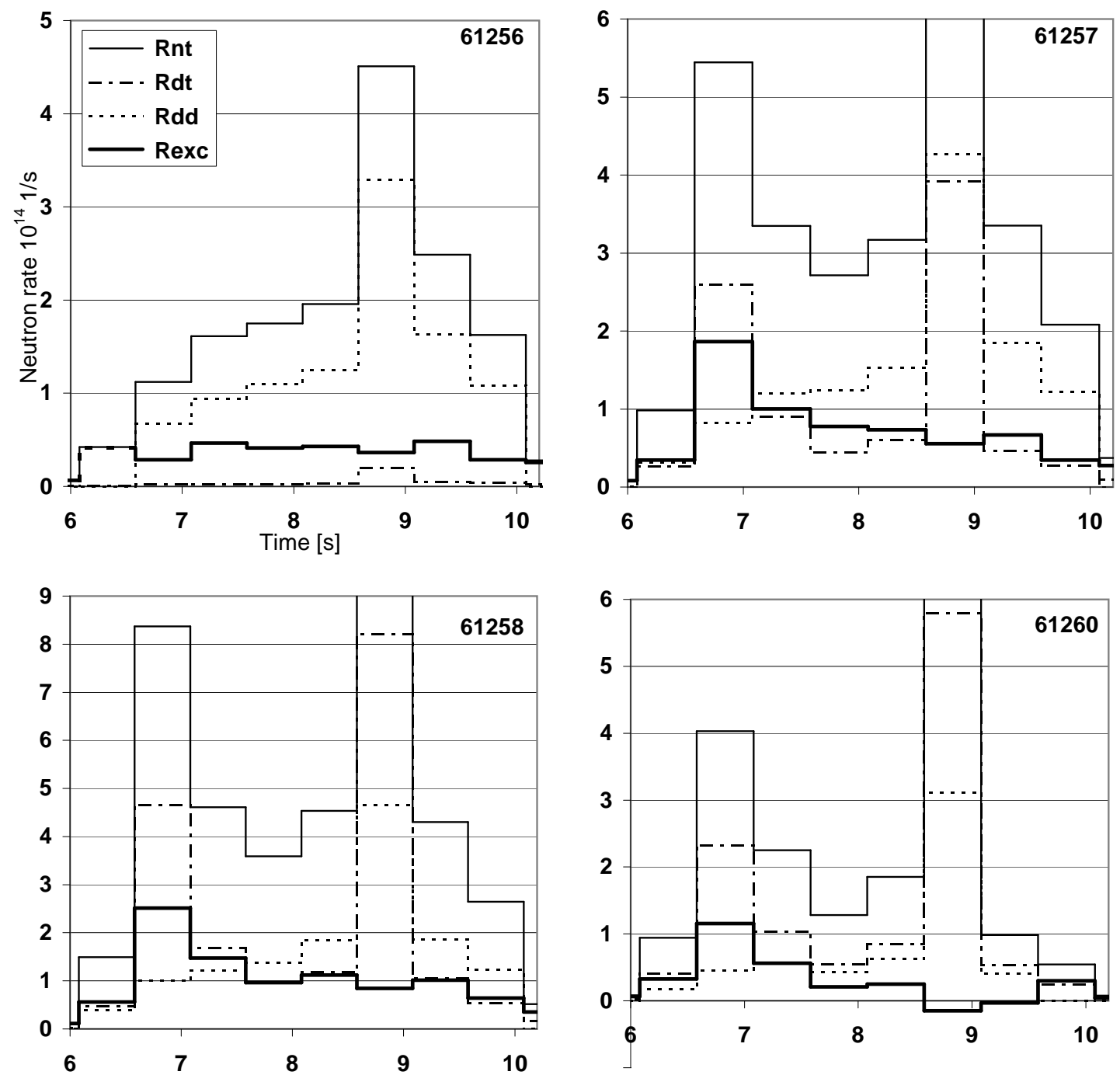

Figure 9. Temporal evolution of neutron rate and the calculated neutron excess during selected pulses at $500 \mathrm{~ms}$ time resolution at which the $R_{\mathrm{DD}}$ data is available. Pulses 61256-61258 are similar with monochromatic heating but with increasing tritium puffing: 0, 3 and $5 \mathrm{mg}$, respectively. Pulse 61260 is polychromatic pulse with $3 \mathrm{mg}$ $\mathrm{T}$ puff. Note how the excess increases (along with DT rate) after T puff at $6.5 \mathrm{~s}$ and then slowly decays. The NBI blip at $8.5 \mathrm{~s}$ is seen as spike in total, DD and DT rate while the excess is expected to be unaffected. For clarity, the tip of total neutron date during the NBI blip is truncated in plot. Due to the systematic errors caused by large DT neutron fraction, $R_{\mathrm{DD}}$ has reduced accuracy in 61260 (DT neutrons about $50 \%$ of total) and possibly momentarily after $\mathrm{T}$ puff in $61257-8$. 

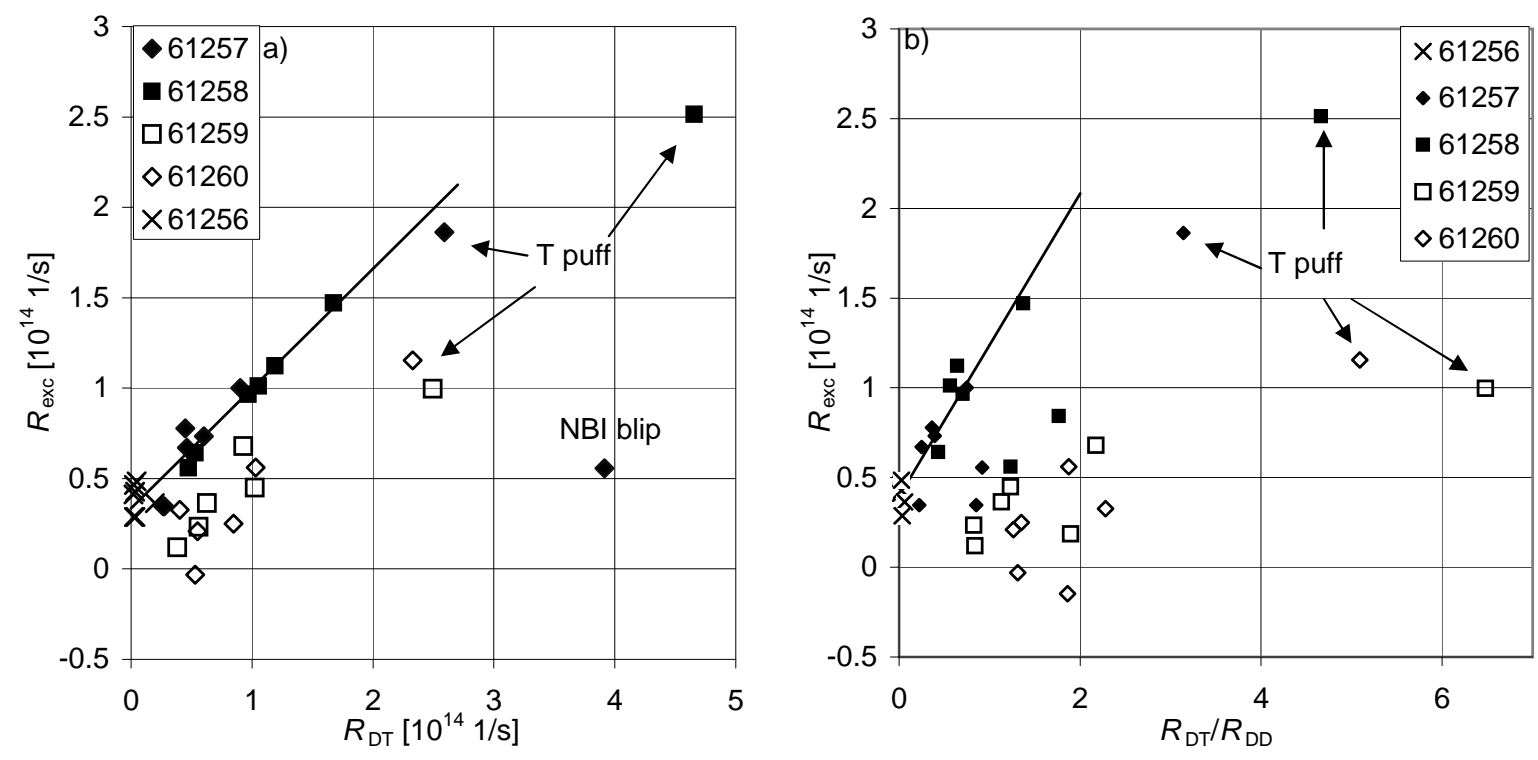

Figure 10. a) The correlation between the DT neutron rate $\left(R_{\mathrm{DT}}\right)$ and the neutron excess $\left(R_{\text {exc }}\right)$. It is seen that in the case of monochromatic heating points lie along a common line (best fit shown). This suggests that the ion temperature and the fast ion distribution are nearly constant and repeatable in these pulses, and $R_{\mathrm{DT}}$ and $R_{\text {exc }}$ scale with varying tritium density. The offset at $R_{\mathrm{DT}}=0$ corresponds to other excess neutron generating mechanisms and the fraction scaling with $R_{\mathrm{DT}}$ can be associated with pT reaction. The points for the polychromatic pulses lie below those of the monochromatic pulses suggesting that the fast ion tail is weaker and the non-pT processes are unimportant. b) Excess neutron rate vs. ratio $R_{\mathrm{DT}} / R_{\mathrm{DD}}$ i. e. a measure of tritium concentration in plasma. Most points from the monochromatic pulses follow a linear dependence indicating that the excess scales linearly with tritium concentration. The outliers arise due to T puffing, NBI blips and early in the pulse during RF ramp-up. Polychromatic pulses again are below monochromatic pulses, as is expected for weaker hot tail.

The spatial distribution of the fast ions in plasma was studied by PION simulations. In Figure 11, we compare the monochromatic (61257) vs. polychromatic (61260) pulses with $3 \mathrm{mg} \mathrm{T}$ puff. As expected, in the monochromatic case, fast ions are seen concentrated close to the centre of plasma while in the polychromatic case the largest population is further away from the centre. The volume-integrated fast ion spectrum shows that the fast ion tail appears hotter and stronger in the monochromatic case. At low energies relevant for the NPA measurements, differences are small, however. The PION simulations also suggest that the ion tail spectrum differs significantly from simple Maxwellian form even locally. These observations are consistent with the small temperature differences seen by NPA while large changes were seen in gamma measurements. However, quantitative comparisons cannot be performed credibly because the absolute results depend very sensitively on the input parameters and experimentally measured input data to PION is not of sufficient quality. 
a)

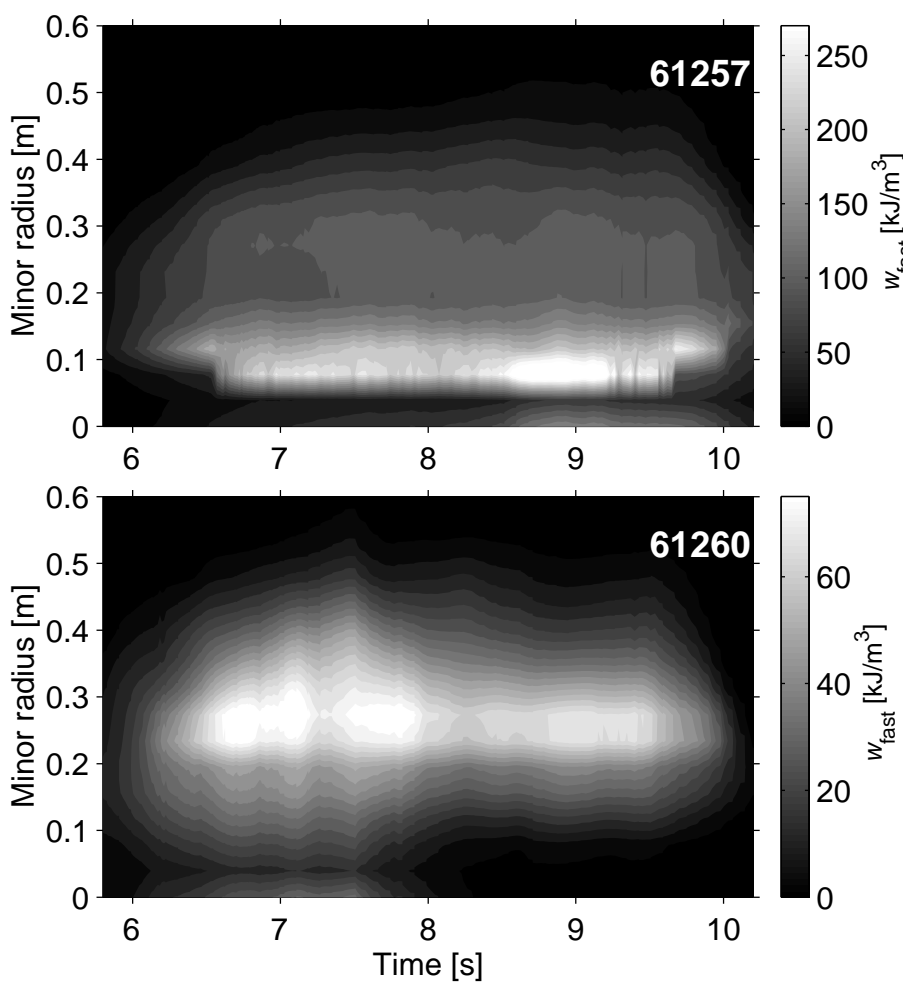

b)

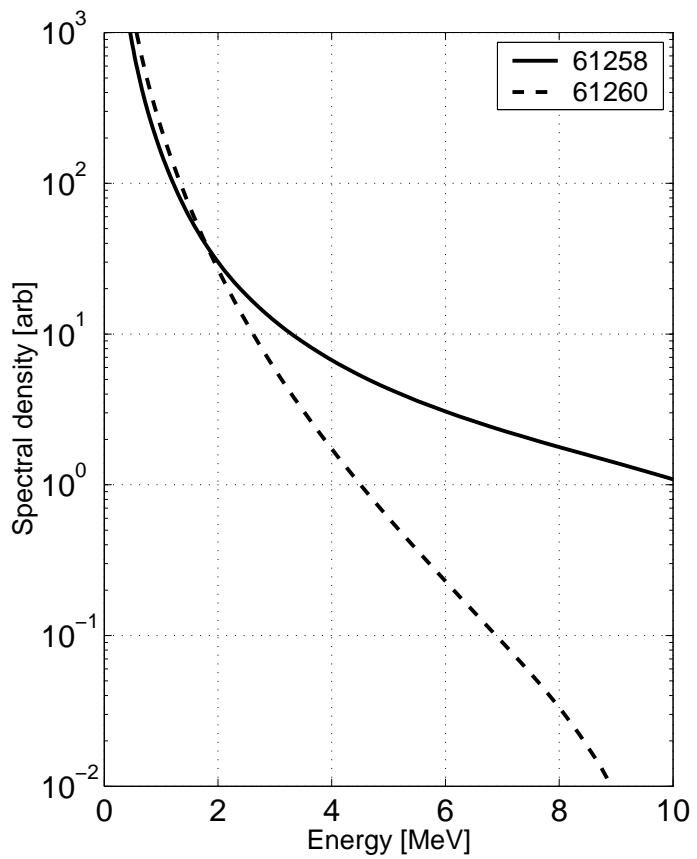

Figure 11. a) Fast ion energy density in PION simulation in case of monochromatic (61257) and polychromatic (61260) heating b) An example volume-integrated fast ion spectra time-averaged over interval 8-8.5 s, just before NBI blip. It should be noted that spectra are very sensitive to input data.

\section{Discussion}

We have shown that the yield of pT reaction can be measured in plasmas of low tritium density, despite limitations in the diagnostic techniques. Measuring a weak, low-energy neutron source against a background of energetic fusion neutrons is fundamentally difficult, as the fusion neutrons also contribute to the low-energy spectrum due to scattering. In the present experiment, the excess neutron production was deduced from the difference between the total neutron production and the sum of the DD and DT neutron production, all measured by different diagnostics. As the DD measurement is disturbed by high DT neutron fraction, good neutron excess data was obtained particularly when the $R_{\mathrm{DT}} / R_{\mathrm{DD}}$ ratio was low. The best conditions existed primarily during the set-up pulses, and pulses 61257 and 61258, apart from the time immediately after tritium puffing. However, even though the DD rate may have error after puffing, the excess yield is also at its maximum so the impact on excess yield is modest.

An 'anomalous' neutron excess was seen in the setup pulses despite very low tritium concentration, with no tritium puffing (but a little recycled tritium from the vessel walls). The likely mechanisms are beam-type fusion induced by RF-accelerated deuterons (partly not detected as DD fusion due to broad neutron spectrum) and 
reactions with plasma impurities (see Sect. 1.2), as this excess was observed when hottest possible ion tail was produced. It is believed that a similar contribution existed also in the monochromatic high-power pulses with tritium puffing. The fact that the other mechanisms do not require tritium suggests that excess neutrons may be produced also in experiments not related to tritium. As the anomalous neutron rate was fairly low, $0.3-0.4 \times 10^{14} 1 / \mathrm{s}$, and heating was optimised to produce hottest possible tail, this could mainly affect other purely RF-minority heated experiments. Without optimal RF-heating, there would not be hot enough ion tail in the plasma and, with neutral beam heating, the DD neutrons would overwhelm the excess neutrons.

Thermal fusion reactivity of the DT reaction is approximately 100 times higher than DD reaction (neutron-producing branch) for typical bulk ion temperatures $(2-3 \mathrm{keV})$ in this experiment. Hence, when $R_{\mathrm{DT}}$ is equal to $R_{\mathrm{DD}}$ triton density is about $1 \%$ of deuteron density, $n_{t} \approx 2 \times 10^{17}$. The slope of the best fit line in Fig. $10 \mathrm{~b}$ (pulses $61256-8$ ) is 0.84 , i. e. this $n_{t}$ corresponds to $\mathrm{pT}$ rate of $0.84 \times 10^{14} 1 / \mathrm{s}$. With total $W_{\text {fast }} \approx 0.6 \mathrm{MJ}$, this corresponds to peak temperature of $580 \mathrm{keV}$ in the integrated parabolic model of fast ion distribution. On one hand this is about $100 \mathrm{keV}$ higher than measured by NPA measurements, on the other hand this is somewhat less that predicted by PION simulations (Fig. 5d). This is consistent with the presence of a very hot ion tail beyond the NPA energy range in these pulses, as suggested by gamma measurements, too. The distribution could be even hotter as the decreased absolute detection efficiency of lowenergy pT neutrons is not taken into account. The companion study has concentrated on correlating pT neutron yield on fast ion temperature and fast ion energy content as measured by the neutral particle analyser [13].

In JET experiments, the fast ion energy content may reach about 1 MJ. If tail temperature was in the range 500-1000 keV with pure RF heating, in 50/50 DT mixture the $\mathrm{pT}$ reaction rate could be on the order of $10^{16} 1 / \mathrm{s}$. This could be a significant fraction of all neutrons if bulk ion temperature remained low. However, when plasma is heated the fusion neutrons would overwhelm the pT neutrons manyfold. The situation could be substantially different in tritium-majority plasmas with very little deuterium present. Under such conditions, neutron rate from deuterium reactions would be low, and pT neutrons could again be a large fraction of the total neutron yield. In principle, pT reaction also is a loss-mechanism to tritium and it consumes energy due to the negative $Q$ value. However, these are insignificant: power lost is about $1 \mathrm{~kW}$ at $10^{16} 1 / \mathrm{s}$, and the fraction of tritons lost in the pT reaction about $10^{-5}$.

Experiments with very large tritium fraction (Full-Tritium Experiment, FTE) are contemplated for JET. The lack of deuterium in plasma would greatly reduce the DT and DD reaction yields. In such plasmas, the T-T fusion reaction (see Fig. 1) is likely to be the dominant thermal neutron source. It produces a continuum spectrum up to $10 \mathrm{MeV}$. If fast proton populations are produced, the $\mathrm{pT}$ reaction rate could be anticipated to be 100 times higher than in TTE conditions (due to higher tritium concentration). The TT reaction rate can be expected to be somewhat smaller than DD rate as $\sigma_{\mathrm{TT}}<\sigma_{D D}$. This would suggest that in experiments producing hot proton tails, the pT neutron yield could 
be a major fraction of the total neutron yield. However, telling the difference between TT and pT neutrons will be difficult as both are overlapping continuum sources.

In addition to the excess neutrons, high energy gammas were observed from the 'true-fusion' branch of the pT reaction: $\mathrm{T}(p, \gamma){ }^{4} \mathrm{He}(Q=19.814 \mathrm{MeV})$. This branch has no threshold but the reaction cross-section is small, less than $0.1 \mathrm{mb}$ for $E_{p}<6 \mathrm{MeV}$ [6], i. e. 3-4 orders of magnitude weaker than the neutron-producing reaction above $1 \mathrm{MeV}$. Due to the small cross-section the signal was quite weak. However, this reaction could be interesting for the study of fast protons in high tritium concentration plasmas because the gamma spectrum depends directly on the proton spectrum. In the energy range near $20 \mathrm{MeV}$, the gamma measurement would be free from background.

\section{Conclusions}

We have performed an experiment at JET to study proton-triton nuclear reaction in purely RF-heated magnetically-confined fusion reactor plasmas having a low $(\approx 1 \%)$ tritium concentration. We have modelled neutron production from pT reaction, calculated dependence of neutron spectrum on proton tail temperature, and shown that the anisotropy of $\mathrm{pT}$ reaction in the center-of-mass frame causes significant changes in the energy spectrum of neutrons. Experimentally, we have demonstrated that pT reaction can be measured also in plasmas having low tritium concentration, despite certain diagnostic limitations. The observed yield is seen to be linearly proportional to tritium density in plasma when the $R_{\mathrm{DT}} / R_{\mathrm{DD}}$ ratio is low, and matches the theoretical modelling well in magnitude.

The pT reaction is likely to have largest impact in future in purely RF-heated experiments with low bulk plasma temperatures and deuterium-poor tritium-majority experiments. In hot plasmas containing major fraction of deuterium, particularly with neutral beam heating, fusion neutrons are likely to overwhelm pT neutrons. In nearly pure tritium plasmas with very hot proton population, neutrons from $\mathrm{pT}$ reaction can be a very significant fraction of all neutrons produced.

\section{Acknowledgements}

This work has been carried out under the European Fusion Development Agreement.

\section{References}

[1] G Audi, A H Wapstra, and C Thibault, Nuclear Physics A 729337 (2003).

[2] M J Mantsinen et al., Nuclear fusion 411815 (2001).

[3] C R Brune, Phys. Rev. C 60015801 (1999).

[4] M Drosg, DROSG-2000, codes and database for 59 neutron source reactions, documented in the IAEA report IAEA-NDS-87 Rev. 8 (January 2003), retrieved online from the IAEA Nuclear Data Section.

[5] R M White, D A Resler and G M Hale "FENDL/C-2.0 Charged particle reaction data library for fusion applications", summary documentation by A B Pashchenko and H Wienke, report 
IAEA-NDS-177, IAEA, March 1997. Data library retrieved online from the IAEA Nuclear Data Section.

[6] K I Hahn, C R Brune, and R W Kavanaugh, Phys. Rev. C 511624 (1995).

[7] M T Swinhoe, O N Jarvis, Nucl. Instrum. Methods in Phys. Research A, 221, 460-465 (1984).

[8] J M Adams, et al., Nucl. Instrum. Methods in Phys. Research A, 329, 277 (1993).

[9] S Conroy, et al., Nucl. Fusion 28, 2127 (1988).

[10] L G Eriksson, T Hellsten, and U Willén, Nuclear Fusion 331037 (1993).

[11] M J Mantsinen, et al., Plasma Phys. Control. Fusion 41843 (1999).

[12] L G Eriksson, et al., Phys. Rev. Lett. 811231 (1998).

[13] D Testa, et al., to be published

\section{Appendix: Derivation of distribution function for anisotropic temperatures}

Let us consider separate distributions for parallel ( $E_{\|}, T_{\|}, \quad 1$ dimension) and perpendicular $\left(E_{\perp}, T_{\perp}, 2\right.$ dimensions) energies:

$$
\begin{aligned}
& f_{\|}\left(E_{\|}\right)=\frac{1}{\sqrt{\pi T_{\|} E_{\|}}} \exp \left(-\frac{E_{\|}}{T_{\|}}\right) \\
& f_{\perp}\left(E_{\perp}\right)=\frac{1}{T_{\perp}} \exp \left(-\frac{E_{\perp}}{T_{\perp}}\right)
\end{aligned}
$$

As these are independent, joint distribution $f\left(E_{\|}, E_{\perp}\right)$ can be defined

$$
\begin{aligned}
& f\left(E_{\|}, E_{\perp}\right)=f_{\|}\left(E_{\|}\right) \times f_{\perp}\left(E_{\perp}\right) \\
& f\left(E_{\|}, E_{\perp}\right)=\frac{1}{\sqrt{\pi T_{\|} T_{\perp}^{2} E_{\|}}} \exp \left(-\frac{E_{\|}}{T_{\|}}\right) \exp \left(-\frac{E_{\perp}}{T_{\perp}}\right)
\end{aligned}
$$

Total particle energy $E=E_{\|}+E_{\perp}$. Fixing total energy, $E_{\|}$and $E_{\perp}$ are no longer independent but $E_{\perp}=E-E_{\|}$. Distribution at energy $E, F(E)$, can be found by integrating over all possible $E_{\|}$:

$$
\begin{aligned}
F(E) & =\int_{0}^{E} f\left(E_{\|}, E-E_{\|}\right) d E_{\|} \\
& =\int_{0}^{E} \frac{1}{\sqrt{\pi T_{\|} T_{\perp}^{2} E_{\|}}} \exp \left(-\frac{E_{\|}}{T_{\|}}\right) \exp \left(-\frac{E-E_{\|}}{T_{\perp}}\right) d E_{\|} \\
& =\frac{1}{\sqrt{\pi T_{\|} T_{\perp}^{2}}} \int_{0}^{E} \frac{1}{\sqrt{E_{\|}}} \exp \left(-\frac{E_{\|}}{T_{\|}}-\frac{E}{T_{\perp}}+\frac{E_{\|}}{T_{\perp}}\right) d E_{\|} \\
& =\frac{1}{\sqrt{\pi T_{\|} T_{\perp}^{2}}} \exp \left(-\frac{E}{T_{\perp}}\right) \int_{0}^{E} \frac{1}{\sqrt{E_{\|}}} \exp \left(-E_{\|}\left(\frac{1}{T_{\|}}-\frac{1}{T_{\perp}}\right)\right) d E_{\|} \\
F(E) & =\frac{1}{\sqrt{\pi T_{\|} T_{\perp}^{2}}} \exp \left(-\frac{E}{T_{\perp}}\right) \int_{0}^{E} \frac{1}{\sqrt{E_{\|}}} \exp \left(-\frac{E_{\|}}{T^{*}}\right) d E_{\|},
\end{aligned}
$$

where $T^{*}$ has been defined as

$$
\frac{1}{T_{\|}}-\frac{1}{T_{\perp}}=\frac{T_{\perp}-T_{\|}}{T_{\|} T_{\perp}} \equiv \frac{1}{T^{*}}
$$


For the integral, it can be found (Mathematica) that

$$
\int \frac{1}{x^{1 / 2}} \exp \left(-\frac{x}{a}\right) d x=\sqrt{\pi a} \operatorname{erf}\left(\sqrt{\frac{x}{a}}\right)
$$

Substituting this,

$$
F(E)=\sqrt{\frac{T^{*}}{T_{\|} T_{\perp}^{2}}} \exp \left(-\frac{E}{T_{\perp}}\right) \operatorname{erf}\left(\sqrt{\frac{E}{T^{*}}}\right)
$$

where identity $\operatorname{erf}(0)=0$ has been used. Now

$$
\sqrt{\frac{T^{*}}{T_{\|} T_{\perp}^{2}}}=\sqrt{\frac{T_{\|} T_{\perp}}{\left(T_{\perp}-T_{\|}\right) T_{\|} T_{\perp}^{2}}}=\frac{1}{\sqrt{\left(T_{\perp}-T_{\|}\right) T_{\perp}}}=\frac{1}{T_{\perp}\left(1-T_{\|} / T_{\perp}\right)^{1 / 2}} .
$$

Using this and the definition of $T^{*}$, one gets the general distribution function for particles having different parallel and perpendicular temperatures:

$$
F(E)=\frac{1}{T_{\perp}\left(1-T_{\|} / T_{\perp}\right)^{1 / 2}} \exp \left(-\frac{E}{T_{\perp}}\right) \operatorname{erf}\left(\sqrt{\frac{E}{T_{\|}}-\frac{E}{T_{\perp}}}\right) .
$$

Some special cases are developed next from Eq. 24.

In the highly anisotropic case $T_{\perp} \gg T_{\|},\left(1-T_{\|} / T_{\perp}\right)^{1 / 2}$ approaches 1 . Likewise the argument of erf becomes large when $E \gg T_{\|}$, and $\lim _{x \rightarrow \infty} \operatorname{erf}(x)=1$. With these approximations, one gets the simple Maxwellian form

$$
F(E)=\frac{1}{T_{\perp}} \exp \left(-\frac{E}{T_{\perp}}\right) .
$$

This form effectively assumes that the parallel motion of particles is unimportant. For finite $T_{\|}$, it should be noted that this form is not applicable at very low energies $E \lesssim T_{\|}$because $\lim _{x \rightarrow 0} \operatorname{erf}(x)=0$. Qualitatively, particles are likely to have some parallel energy due to the finite $T_{\|}$(neglected in Eq. 25), which decreases the probability of finding particles at very low total energies.

For moderately high anisotropy, Eq. 25 can be refined by retaining the factor depending on both $T_{\perp}$ and $T_{\|}$:

$$
F(E)=\frac{1}{T_{\perp}\left(1-T_{\|} / T_{\perp}\right)^{1 / 2}} \exp \left(-\frac{E}{T_{\perp}}\right)
$$

In the limit of slightly anisotropic distributions $\left(T_{\|} \approx T_{\perp}\right), T^{*}$ becomes large, and the argument of erf in Eq. 22, becomes small. erf can then be replaced by the 1st term of its Taylor expansion, $\operatorname{erf}(x) \approx 2 / \sqrt{\pi} x$. Eq. 22 can then be expressed as

$$
\begin{aligned}
& F(E)=\sqrt{\frac{T^{*}}{T_{\|} T_{\perp}^{2}}} \exp \left(-\frac{E}{T_{\perp}}\right) \frac{2}{\sqrt{\pi}} \sqrt{\frac{E}{T^{*}}} \\
& F(E)=\frac{2 \sqrt{E}}{\sqrt{\pi T_{\|}} T_{\perp}} \exp \left(-\frac{E}{T_{\perp}}\right) .
\end{aligned}
$$


Eq. 28 is only valid when $T_{\|}$and $T_{\perp}$ differ only slightly $\left(\approx T_{\|} / T_{\perp}<0.8\right)$.

In fully isotropic case, $T=T_{\|}=T_{\perp}$. Substituting this into Eq. 28, one obtains

$$
F(E)=\frac{2 \sqrt{E}}{\sqrt{\pi T^{3}}} \exp \left(-\frac{E}{T}\right)
$$

This is the standard equation for gas-kinetic energy distribtion (i. e. isotropic, fully 3-dimensional case). 